(c) 2013 IEEE. Personal use of this material is permitted. Permission from IEEE must be obtained for all other users, including reprinting/ republishing this material for advertising or promotional purposes, creating new collective works for resale or redistribution to servers or lists, or reuse of any copyrighted components of this work in other works. DOI:

10.1109/JSEN.2012.2217955

\title{
Evaluation of Electrical Capacitance Tomography Sensors for Concentric Annulus
}

\author{
Jiamin Ye, Member, IEEE, and Wuqiang Yang, Fellow, IEEE
}

\begin{abstract}
Electrical capacitance tomography (ECT) sensor is commonly used with circular or square pipes or vessels. In this paper, an ECT sensor of concentric annulus shape is presented. To investigate the effect of the configuration of electrodes and the measuring strategy on the quality of reconstructed images, the ECT sensors with internal-external electrodes (IEE) and external electrodes (EE) are investigated. For the IEE sensor, six different measuring strategies are considered. The capacitance between different electrode pairs is calculated for some typical permittivity distributions using a finite element method (FEM). The obtained capacitance data are then used to reconstruct images using Landweber iteration algorithm. The sensitivity distributions for the IEE and EE sensors are analysed. Simulation results show that the IEE sensor with 12 external electrodes and 4 internal electrodes combining with an external-opposite strategy can reconstruct good images for most permittivity distributions.
\end{abstract}

Index Terms-Electrical capacitance tomography, capacitance sensor, internal-external electrode, image reconstruction, concentric annulus.

\section{INTRODUCTION}

As a visualization technique for dielectric processes, electrical capacitance tomography (ECT) has been widely used in different fields, e.g. gas/solid conveying systems and pneumatic fluidised beds [1]-[4].

In general, 8 or 12 electrodes are mounted outside a pipe to obtain capacitance measurements [5]. The images reconstructed by a traditional ECT sensor with external electrodes (EE) are usually blurred in the central areas. To improve the image quality for a circulating fluidised bed (CFB), Liu et al [6] presented a square ECT sensor with internal-external electrodes (IEE). The IEE sensor effectively increases the relative sensitivity in the middle of the sensing area [6]. Rezvanpour et al [7] recently designed an ECT sensor with circular internal-external electrodes to investigate the droplet distribution in electrohydrodynamic atomization.

Manuscript received $\mathrm{xx}, \mathrm{xx}, \mathrm{xxxx}$.

J. Ye is with the School of Process, Environmental and Materials Engineering, University of Leeds, Leeds LS2 9JT, U.K. (e-mail: yejiamin@ hotmail.com ).

W. Yang is with the School of Electrical and Electronic Engineering, University of Manchester, Manchester M13 9PL, U.K. (e-mail: wuqiang.yang@ manchester.ac.uk ).
For some multiphase flow systems, e.g. heat exchangers, nuclear reactor cores, combustion systems [8] and drilling or oil extraction in petroleum industry [9], the flow area is often a concentric annulus. Meanwhile, the flow in an annulus channel is capable of replicating some of the phenomena encountered in the complex geometries [10].

To investigate the distribution of the fluid in an annulus area using ECT, a possibility is to use an IEE sensor. The effect of the number of electrodes in ECT senor was evaluated by Peng et al [11]. However, the performance of IEE sensors for the concentric annulus has not been studied. Meanwhile, the number of the internal and external electrodes and the measuring strategy should be discussed too.

In this paper, five ECT sensors with different number of external and internal electrodes combining with six measuring strategies are investigated by comparing with traditional ECT sensors.

In the following, an ECT sensor with external electrodes only is called "EE sensor", and an ECT sensor with internal and external electrodes is called "IEE sensor".

\section{COMPARISON OF ECT SENSORS WITH DIFFERENT ELECTRODE STRUCTURE}

\section{A. ECT Sensor}

Fig. 1 shows a typical IEE sensor and a typical EE sensor used in this work. Fig. 1(a) is an ECT sensor with 8 external electrodes and 4 internal electrodes and Fig. 1(b) is a traditional ECT sensor with 8 external electrodes. The ratio of the electrode angle and the gap angle is 9:1. The ratio of the inner radius and outer radius of the internal pipe, the inner radius and outer radius of the external pipe, and the screen radius is 0.3:0.4:1:1.1:1.2.

The main conclusion of [11] was that a 12-electrode EE ECT sensor should be recommended for most applications. In this research, to compare the effect of the electrode configuration, two EE ECT sensors with 12 and 8 electrodes and five IEE ECT sensors with different number of external-internal electrodes, $12-12,12-8,12-4,8-8$ and 8-4, are investigated.

\section{B. Measuring Strategy}

To determine the measuring strategy, the normalised sensitivity distributions (the definition of the sensitivity will be 
given in the next section) between different electrode pairs for an IEE sensor are illustrated in Fig. 2. It can be seen that the areas between different opposite electrode pairs with a higher sensitivity can cover the whole sensing area just like the external electrodes. Meanwhile, the sensitivities between different internal electrode pairs also have a certain influence on the sensing area. In this paper, six different measuring strategies, including external-internal (EI) strategy, externalopposite-internal (EOI) strategy, opposite (O) strategy, external-opposite (EO) strategy, opposite-internal (OI) strategy

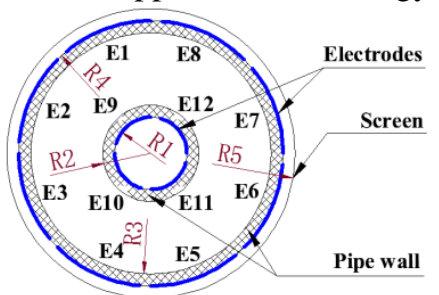

(a)

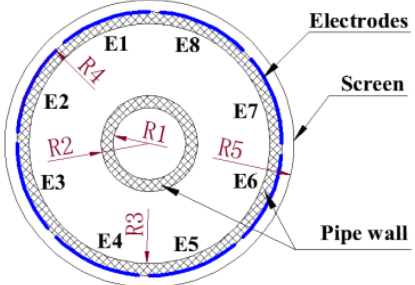

(b)
Fig. 1 ECT sensors for concentric annulus, R1:R2:R3:R4:R5 = 0.3:0.4:1: 1.1:1.2. (a)IEE sensor. (b)EE sensor.

TABLE I Independent number of measurements of different measuring strategies.

\begin{tabular}{lll}
\hline \hline Sensor & Measuring strategy & Number of measured capacitances \\
\hline & External-Internal (EI) & $N_{E} \cdot\left(N_{E}-1\right) / 2+N_{I} \cdot\left(N_{I}-1\right) / 2$ \\
& External-Opposite-Internal(EOI) & $\left(N_{E}+N_{I}\right) \cdot\left(N_{E}+N_{I}-1\right) / 2$ \\
& Opposite (O) & $N_{E} \cdot N_{I}$ \\
IEE & External-Opposite (EO) & $N_{E} \cdot\left(N_{E}-1\right) / 2+N_{E} \cdot N_{I}$ \\
& Opposite-Internal(OI) & $N_{E} \cdot N_{I}+N_{I} \cdot\left(N_{I}-1\right) / 2$ \\
& External (E) & $N_{E} \cdot\left(N_{E}-1\right) / 2$ \\
\hline EE & External & $N_{E} \cdot\left(N_{E}-1\right) / 2$ \\
\hline \hline
\end{tabular}

and external (E) strategy are considered.

A traditional measuring procedure for an EE sensor is as follows. E1 is selected as an excitation electrode and the capacitances between $\mathrm{E} 1$ and other electrodes, represented by $C_{1,2}, C_{1,3}, \cdots \cdots$, and $C_{1,8}$, are measured. Then E2 is selected as an excitation electrode and the capacitances $C_{2,3}$, $C_{2,4}, \cdots \cdots$, and $C_{2,8}$ are measured. This process continues until $C_{7,8}$ is obtained. The total number of measured capacitances is

$$
M=N_{E} \cdot\left(N_{E}-1\right) / 2
$$

where $N_{E}$ is the number of external electrodes. For example, $N_{E}=8$ and $M=28$. The measuring procedure for the internal electrodes is the same as for the EE sensor.

The measuring procedure for opposite electrodes is as follows. E1 is selected as an excitation electrode and the capacitances between E1 and the internal electrodes, represented by $C_{1,9}, C_{1,10}, \cdots \cdots$, and $C_{1,12}$, are measured. Then E2 is selected as an excitation electrode and the capacitances $C_{2,9}, C_{2,10}, \cdots \cdots$, and $C_{2,12}$ are measured. This process continues until $C_{8,12}$ is obtained. The total number of measured capacitances is

$$
M=N_{E} \cdot N_{I}
$$

where $N_{I}$ is the number of internal electrodes. For example, $N_{I}=4$ and $M=32$. TABLE I shows the number of independent measurements of different measuring strategies.

After the capacitances between all possible electrode pairs are obtained, the permittivity distribution inside the ECT sensor can be reconstructed using a certain image reconstruction algorithm [13].

\section{Image Reconstruction Algorithm}

Among many reported image reconstruction algorithms, LBP [4] and Landweber iteration [12] are the most popular. The LBP is fast but can only generate qualitative images. The Landweber iteration can provide quantitative image reconstruction with a higher quality [13], but it is slow.

The Landweber iteration can be described as

$$
\mathbf{g}_{l+1}=G\left[\mathbf{g}_{l}-\alpha \mathbf{J}^{T}\left(\mathbf{J} \cdot \mathbf{g}_{l}-\lambda\right)\right]
$$

where $\mathbf{g}$ is the normalised permittivity vector, $\boldsymbol{\lambda}$ is the normalised change in the measured capacitance, $\mathbf{J}$ is the normalised sensitivity matrix, $\alpha$ is the relaxation factor, $l$ is the index of iteration steps and $G$ is the projection operator given by

$$
G[f(x)]= \begin{cases}0 & f(x)<0 \\ f(x) & 0 \leq f(x) \leq 1 \\ 1 & f(x)>1\end{cases}
$$

The initial distribution used for Landweber iteration can be obtained using the LBP algorithm. It can be described as

$$
\mathbf{g}=\frac{\mathbf{J}^{T} \lambda}{\mathbf{J}^{T} \mathbf{I}_{\lambda}} \quad \mathbf{I}_{\lambda}=[1,1, \cdots, 1]
$$

where $\mathbf{I}_{\lambda}$ is an identity vector. The division of the two vectors $\mathbf{J}^{T} \lambda$ and $\mathbf{J}^{T} \mathbf{I}_{\lambda}$ is defined as one numerator component being divided by the corresponding denominator component. Equation (6) is normally used to calculate a sensitivity map as part of the sensitivity matrix.

$$
S_{i j}(x, y)=-\frac{\iint_{p(x, y)} \nabla \phi_{i}(x, y) \cdot \nabla \phi_{j}(x, y) d x d y}{V_{i} \cdot V_{j}}
$$

where $S_{i j}(x, y)$ defines the sensitivity between the $i^{\text {th }}$ electrode and the $j^{\text {th }}$ electrode over the area $p(x, y), \phi_{i}(x, y)$ is the potential distribution inside the sensing domain while the $i^{\text {th }}$ electrode is selected as an excitation electrode by applying a voltage $V_{i}$ on it and the other electrodes are used as detection electrodes. 
In this work, the Landweber iteration is used for image reconstruction.

\section{Simulation Procedure}

To compare the image quality with ECT sensors with different type of electrodes and measuring strategies, some

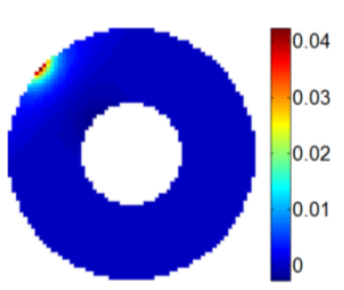

(a)

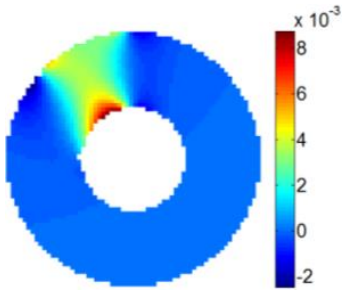

(e)

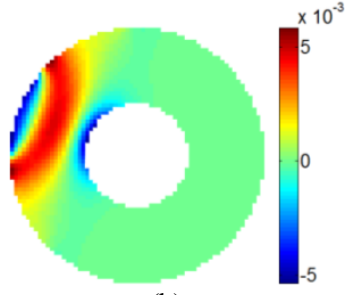

(b)

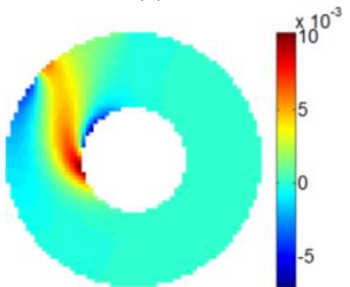

(f)

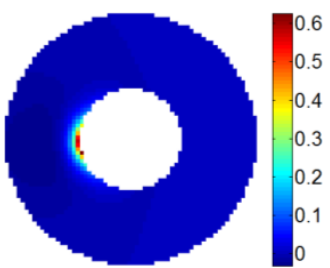

(i)

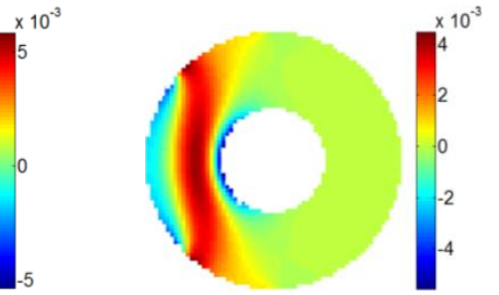

(c)

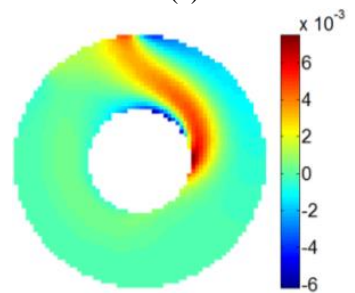

(g)

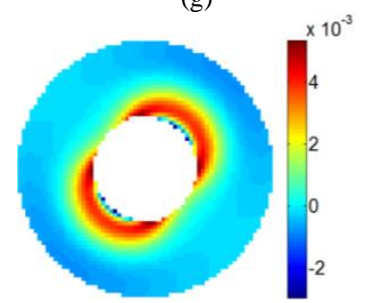

(j)

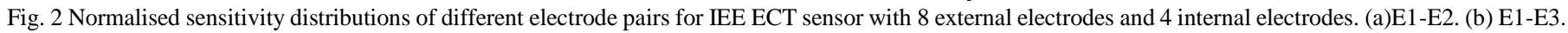
(c) E1-E4. (d) E1-E5. (e) E1-E9. (f) E1-E10. (g) E1_E11. (h) E1-E12. (i) E9-E10. (j) E9-E11.

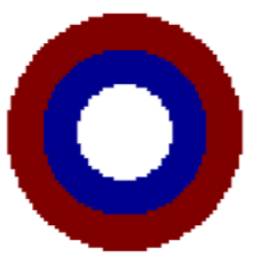

(a)

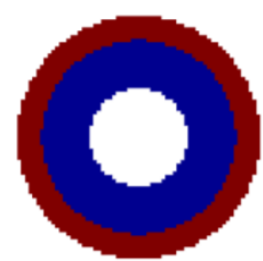

(b)

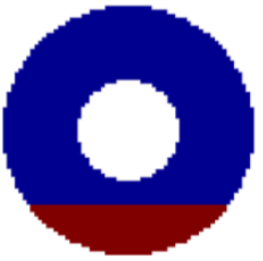

(c)

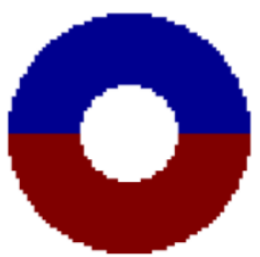

(d)

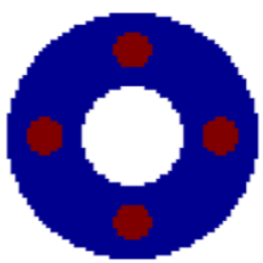

(e)

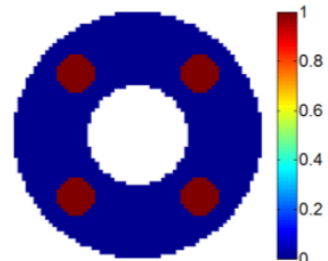

(f)

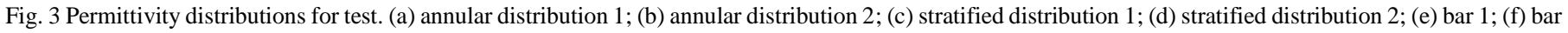
2.

on Landweber iteration were carried out using COMSOL Multiphysics ${ }^{\mathrm{TM}}$ and MatLab ${ }^{\mathrm{TM}}$.

The sensing domain is divided into fine meshes to achieve accurate calculation. The imaging area is divided into $64 \times 64$ grids, which results in 2704 pixels inside the sensor. To assess the quality of image reconstruction, the relative image error and the correlation coefficient between the true permittivity distribution and the reconstructed images are used as criteria. The definition of the relative image error and the correlation coefficient can be seen in (7) and (8), respectively. The lower relative image error and the higher correlation coefficient mean good image reconstruction results.

$$
\text { Image error }=\frac{\|\hat{\mathbf{g}}-\mathbf{g}\|}{\|\mathbf{g}\|} \times 100 \%
$$

$$
\text { Correlation coefficient }=\frac{\sum_{i=1}^{N_{p}}\left(\hat{g}_{i}-\overline{\mathbf{g}}\right)\left(g_{i}-\overline{\mathbf{g}}\right)}{\sqrt{\sum_{i=1}^{N_{p}}\left(\hat{g}_{i}-\overline{\hat{\mathbf{g}}}\right)^{2} \sum_{i=1}^{N_{p}}\left(g_{i}-\overline{\mathbf{g}}\right)^{2}}}
$$

where $\mathbf{g}$ is the normalised true permittivity distribution, $\hat{\mathbf{g}}$ is

the normalised estimated permittivity distribution, i.e. reconstructed image, $N_{p}$ the number of the pixels in the sensing domain. In this work, $N_{p}=2704$.

In the Landweber iteration, the step of iterations plays an important role in image reconstruction for ECT. Peng et al [14] pointed out that the Landweber iteration has a property of so-called semi-convergence, i.e. the image error will decrease first and then increase with the increase of the step of iterations. In this research, the iteration will be stopped while the image 
error with the current iteration step becomes larger than the image error with the previous iteration step [11].

\section{RESULTS AND DISCUSSION}

A. With Landweber Iteration

$N_{\mathrm{E}}$

- IEE-EI IEE-EOI IEE-O IEE-EO IEE-OI IEE-E EE $N_{\mathrm{I}}$
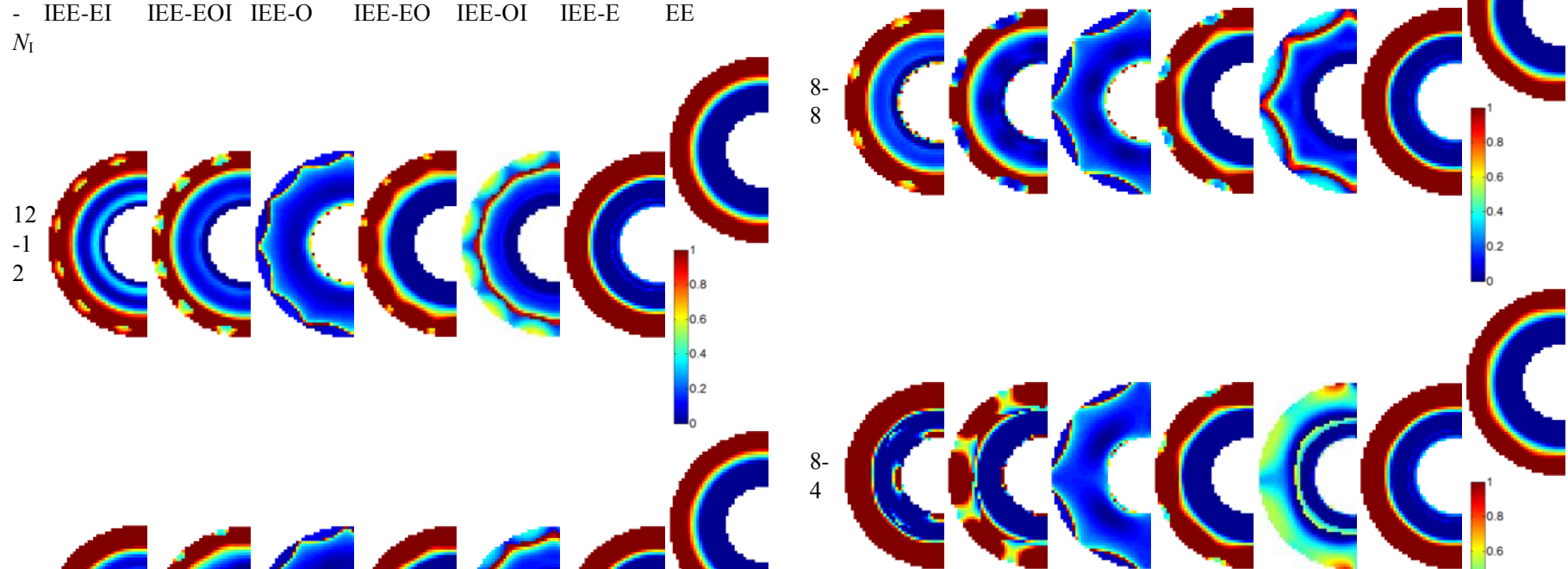

12
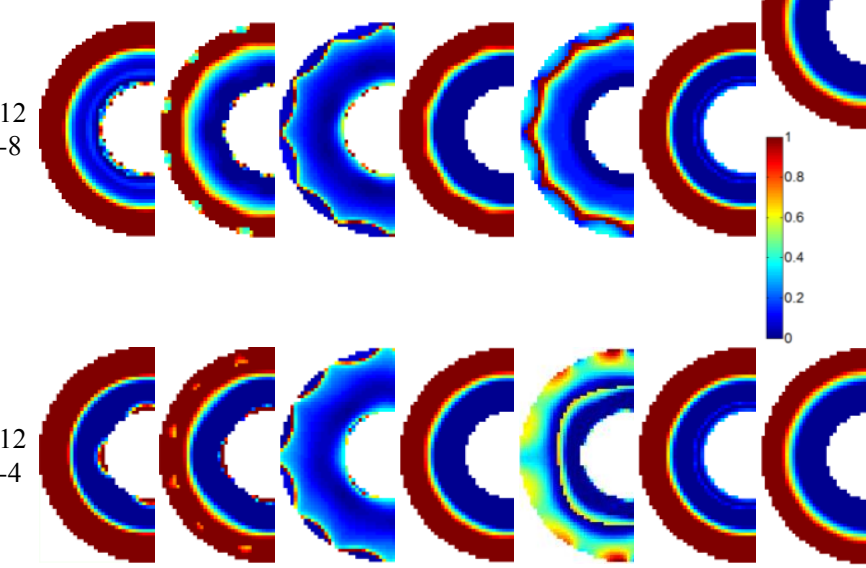

$N_{\text {E- }} N_{\text {I IEE-EI }}$

$12-12$

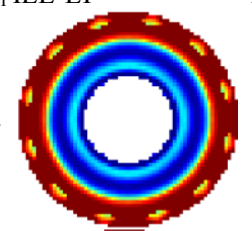

IEE-EOI

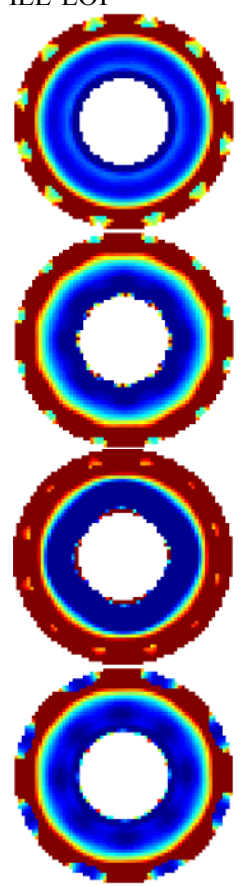

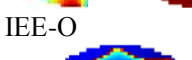
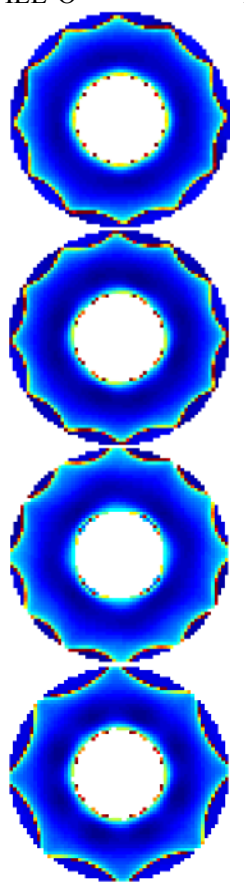

IEE-EO

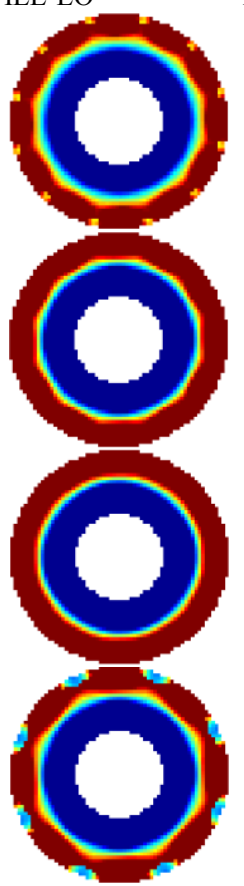

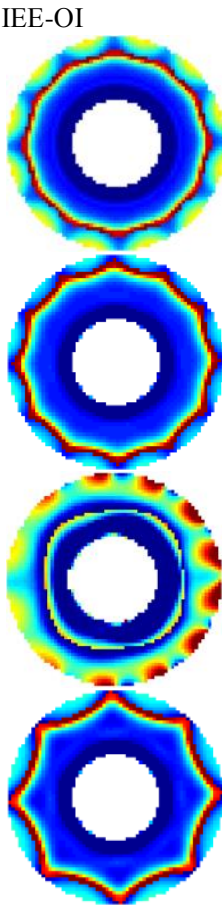
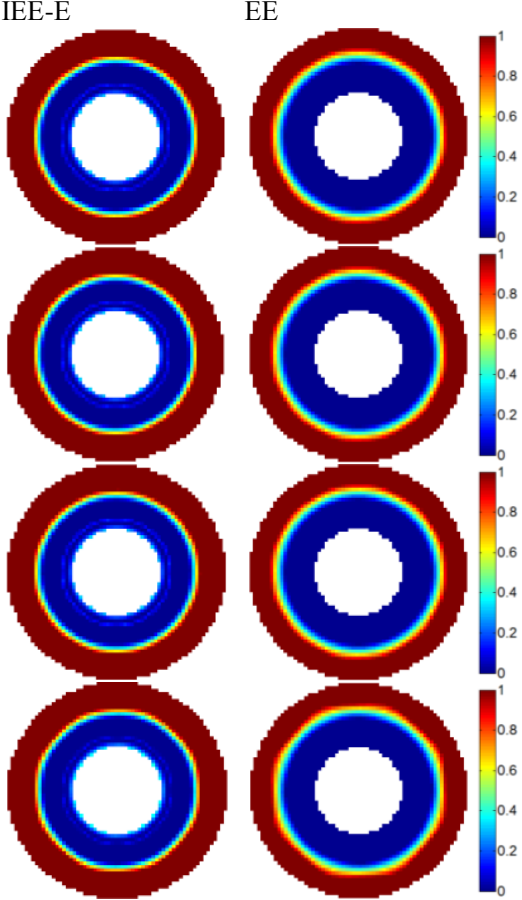


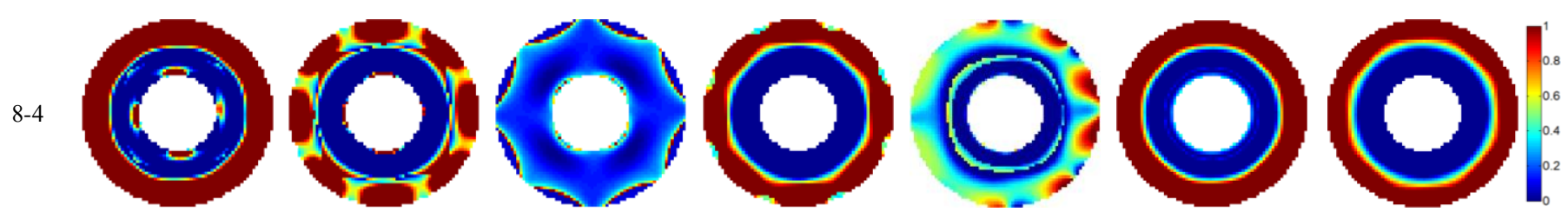

Fig. 4 Images reconstructed for annular distribution 1.

TABLE II Image errors for annular distribution 1.

\begin{tabular}{llllllll}
\hline \hline$N_{\text {E- }} N_{\mathrm{I}}$ & \multicolumn{1}{l}{ IEE-EI } & IEE-EOI & IEE-O & IEE-EO & IEE-OI & IEE-E & EE \\
\hline $12-12$ & 0.2989 & 0.4027 & 0.8014 & 0.2633 & 0.5912 & 0.2006 & 0.2792 \\
$12-8$ & 0.2687 & 0.3502 & 0.7982 & 0.2332 & 0.6359 & 0.2008 & 0.2792 \\
$12-4$ & 0.2343 & 0.2795 & 0.7679 & 0.2360 & 0.6320 & 0.2015 & 0.2792 \\
$8-8$ & 0.3221 & 0.4827 & 0.8013 & 0.3298 & 0.6400 & 0.2002 & 0.2882 \\
$8-4$ & 0.2599 & 0.3563 & 0.7811 & 0.2868 & 0.6072 & 0.2009 & 0.2882 \\
\hline \hline
\end{tabular}

From the reconstructed images in $N_{\mathrm{E}}$

- IEE-EI IEE-EOI IEE-O IEE-EO IEE-OI IEE-E EE $N_{\text {I }}$
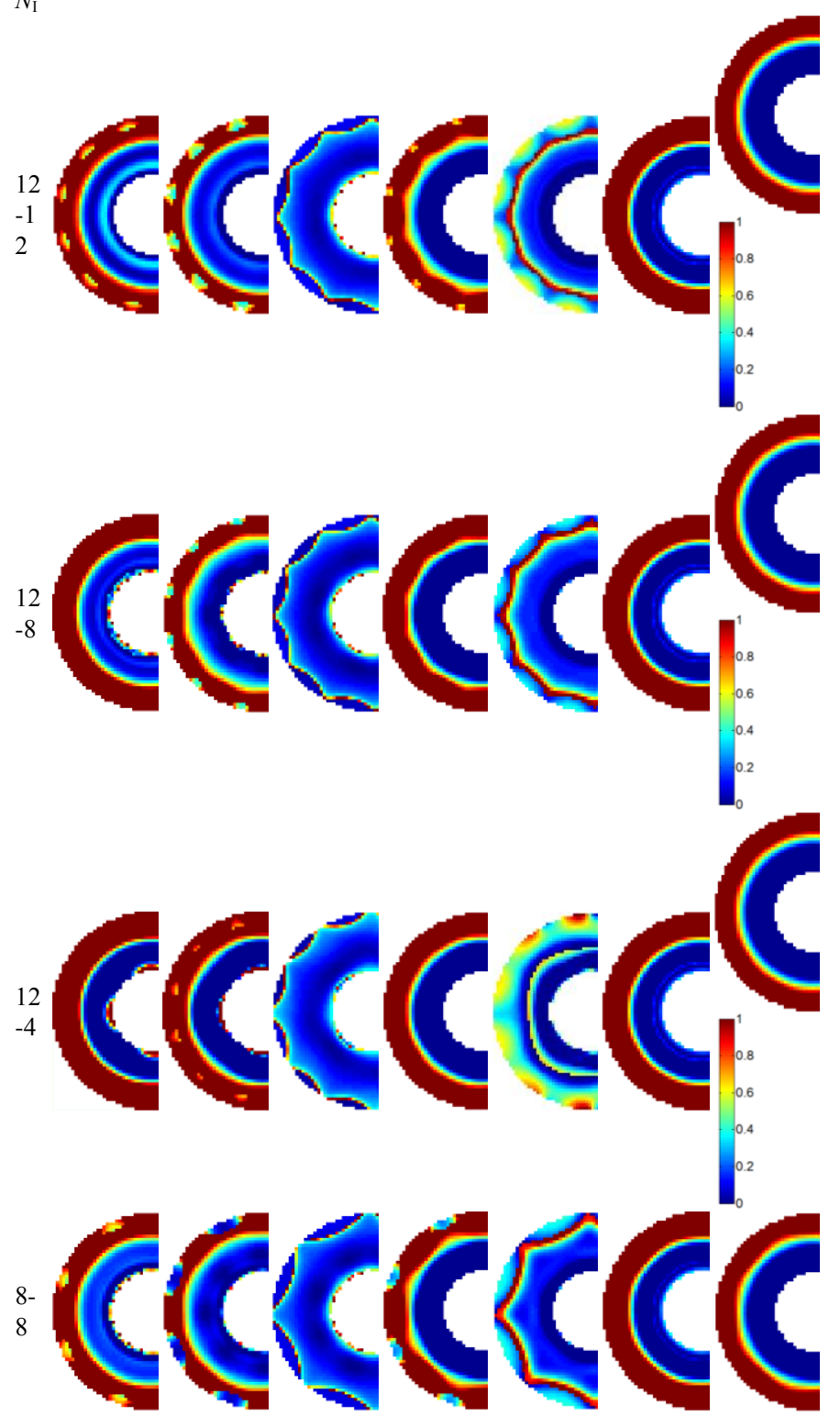

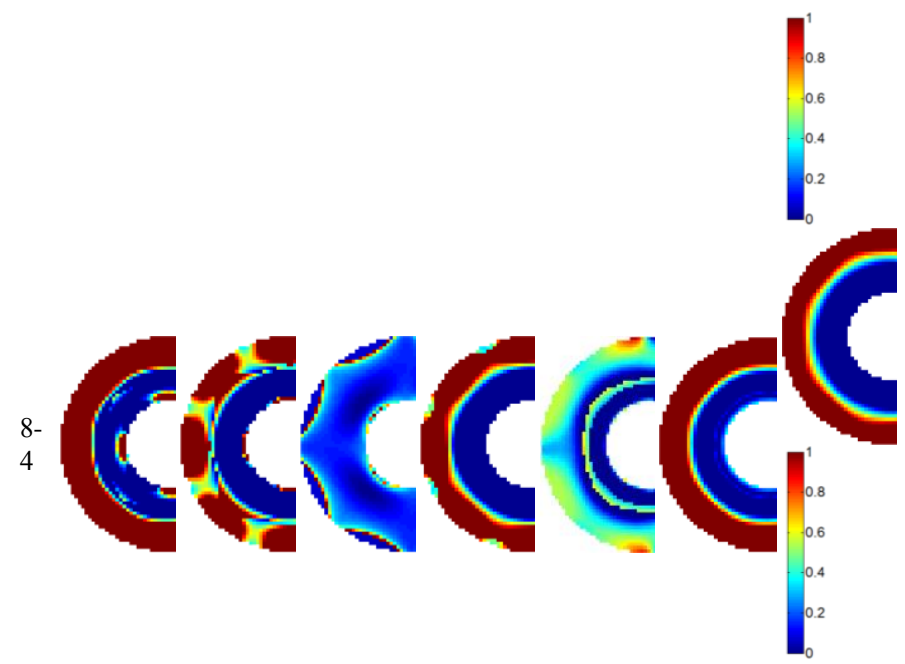

Fig. 4, it can be seen that the images reconstructed by the IEE sensors with the opposite and opposite-internal strategies have serious distortions. The images reconstructed by the IEE sensors combining with the external-internal, external-opposite-internal and external strategies have artifacts near the internal or external pipe wall.

Comparing the results obtained from the Landweber iteration with IEE and EE sensors quantitatively, it can be seen that the quality of the images reconstructed by the IEE sensor with 12 external electrodes is normally better than that with 8 external electrodes. The quality of the images reconstructed by the IEE sensor with 12 external electrodes and 8 or 4 internal electrodes combining with the external-opposite strategy is better than that by the EE sensor with 12 external electrodes. However, the image reconstructed by the IEE sensor with 12 external electrodes and 8 internal electrodes combining with the external-opposite strategy has artifacts near the phase interface.

In summary, the IEE sensor with 12 external electrodes and 4 TABLE III Correlation coefficients for annular distribution 1.

\begin{tabular}{llllllll}
\hline \hline$N_{\text {E- }} N_{\mathrm{I}}$ & IEE-EI & IEE-EOI IEE-O & IEE-EO & IEE-OI & IEE-E & EE \\
\hline $12-12$ & 0.8822 & 0.7981 & 0.2188 & 0.9185 & 0.7045 & 0.9483 & 0.9096 \\
$12-8$ & 0.9044 & 0.8417 & 0.2253 & 0.9346 & 0.6018 & 0.9481 & 0.9096 \\
$12-4$ & 0.9263 & 0.8971 & 0.3136 & 0.9333 & 0.5519 & 0.9478 & 0.9096 \\
$8-8$ & 0.8581 & 0.7222 & 0.2167 & 0.8800 & 0.5956 & 0.9483 & 0.9045 \\
$8-4$ & 0.9090 & 0.8460 & 0.2685 & 0.9055 & 0.6514 & 0.9479 & 0.9045 \\
\hline \hline
\end{tabular}

internal electrodes combining with the external-opposite strategy gives better image reconstruction for annular distribution 1.

$N_{\text {E }}$
- 

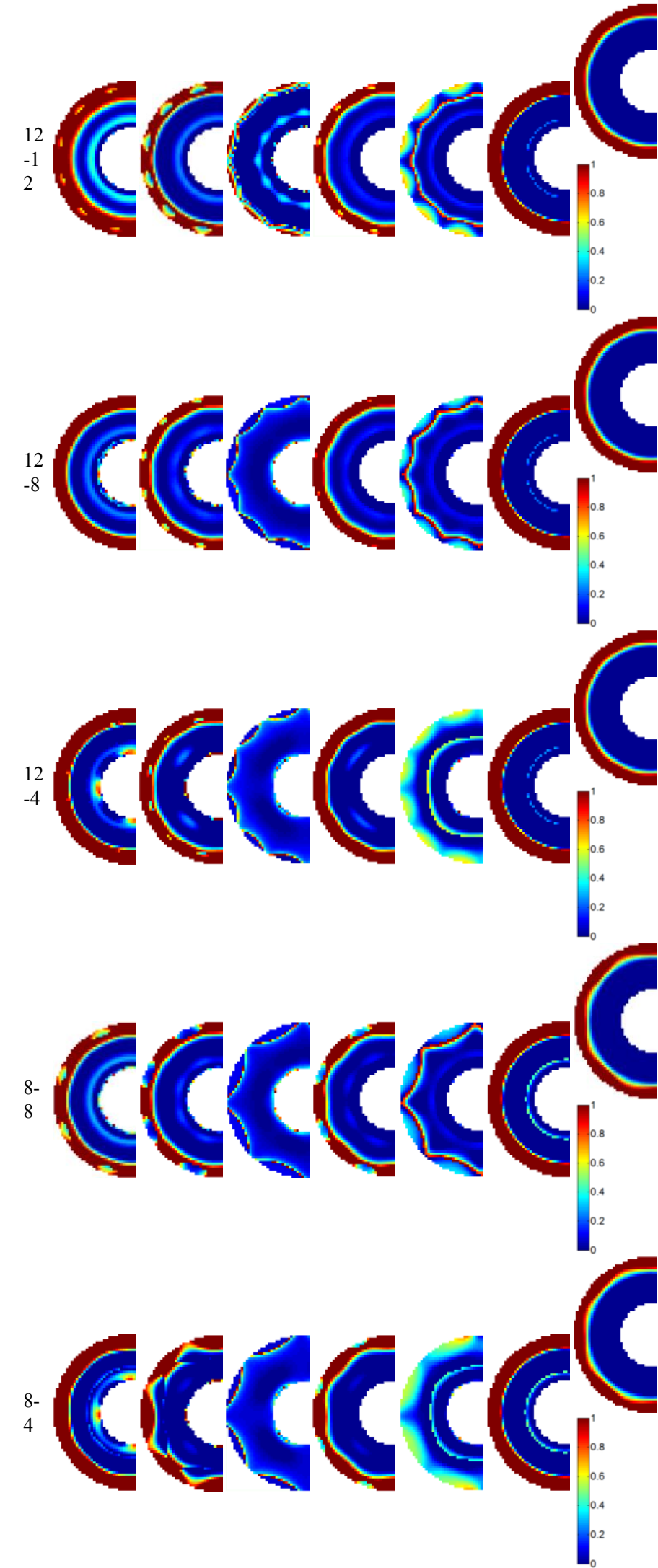

Fig. 5 shows the images reconstructed by the Landweber iteration for annular distribution 2. The relative image errors and correlation coefficients between the true distribution and the reconstructed images are given in TABLE IV and TABLE $\mathrm{V}$, respectively.

From the reconstructed images in - IEE-EI IEE-EOI IEE-O IEE-EO IEE-OI IEE-E EE $N_{\mathrm{I}}$
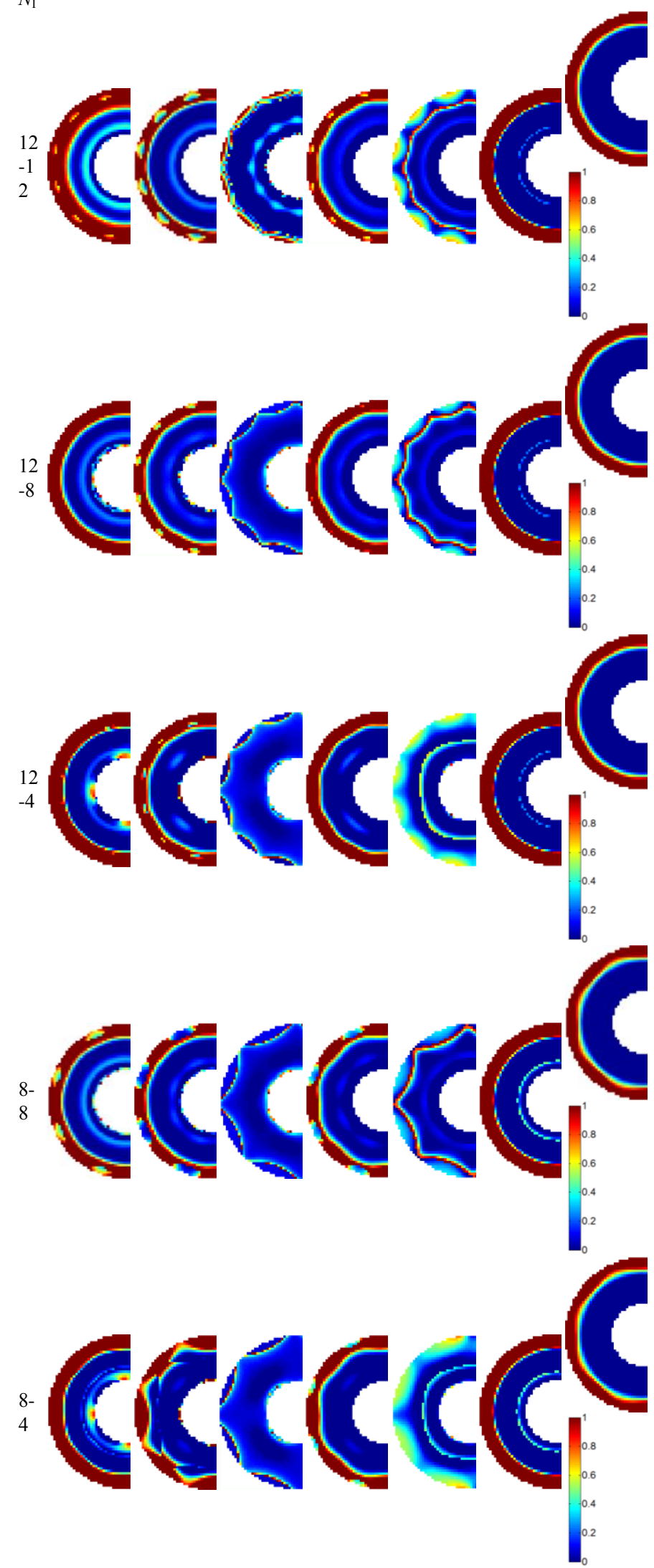

Fig. 5, it can be seen that the images reconstructed by the IEE sensors with the opposite and opposite-internal strategies have 
serious distortions. The images reconstructed by the IEE sensors combining with the external-internal, external-opposite-internal and external strategies have artifacts near the internal or external pipe wall.

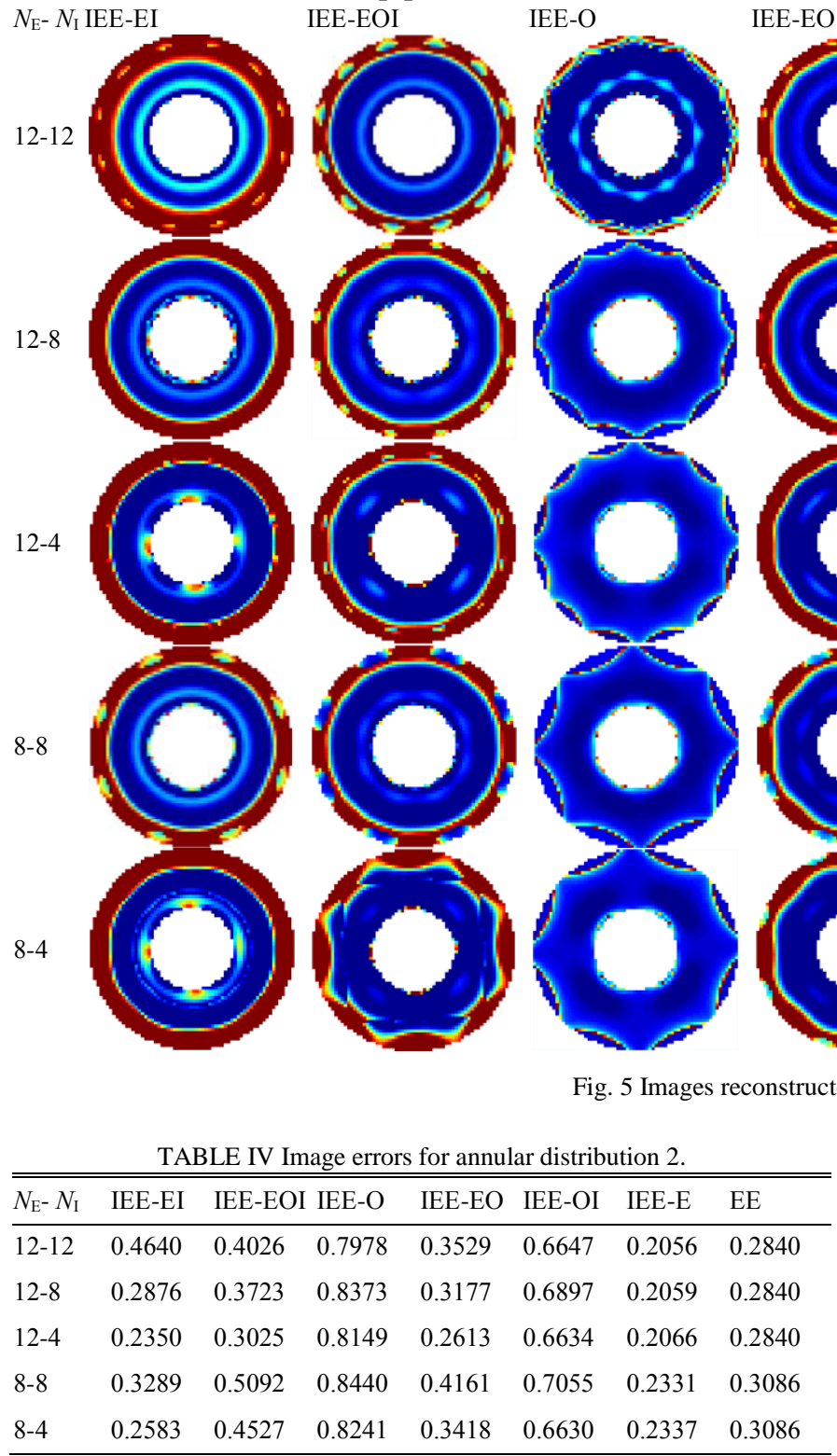

electrodes. The image reconstructed by the IEE sensor with 12 external electrodes and 4 internal electrodes combining with the external-opposite strategy has a lower image error and a higher correlation coefficient than that by the EE sensor with the same number of external electrodes.

In summary, the IEE sensor with 12 external electrodes and 4 internal electrodes combining with the external-opposite strategy gives better image reconstruction for annular distribution 2.

$N_{\mathrm{E}}$

- IEE-EI IEE-EOI IEE-O IEE-EO IEE-OI IEE-E EE $N_{\mathrm{I}}$
Comparing the results obtained from the Landweber iteration with IEE and EE sensors quantitatively, it can be seen that the quality of the images reconstructed by the IEE sensor with 12 external electrodes is normally better than that with 8 external IEE-OI IEE-E EE
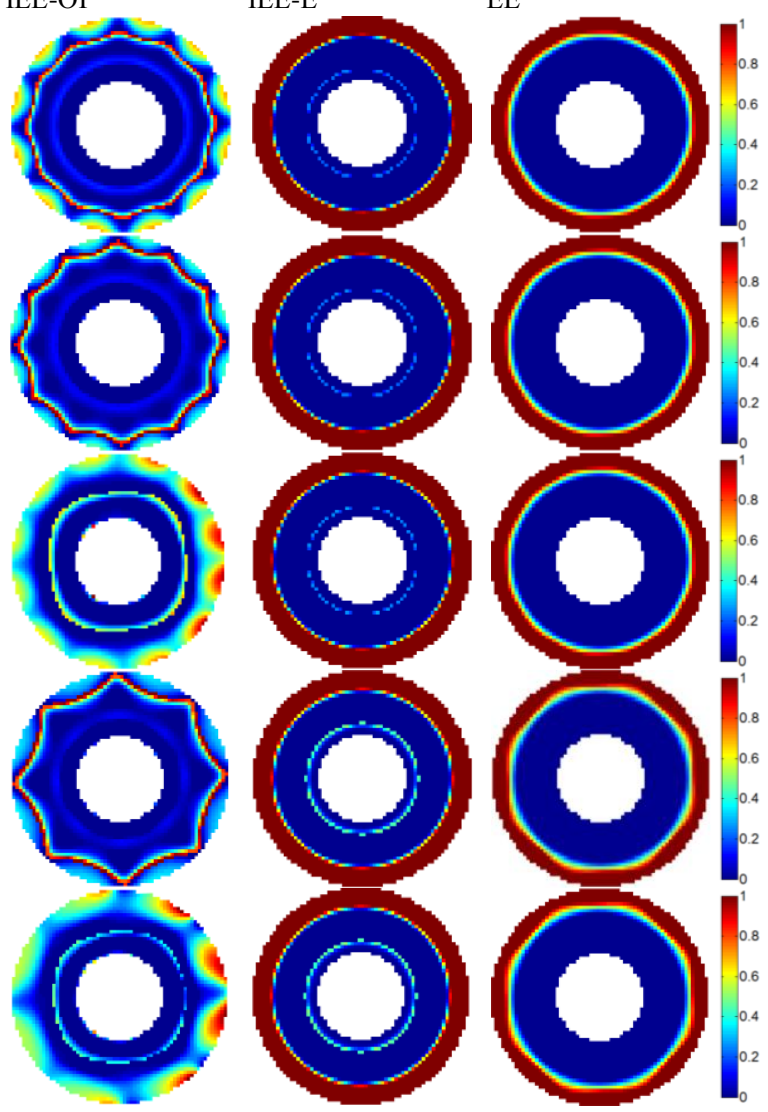

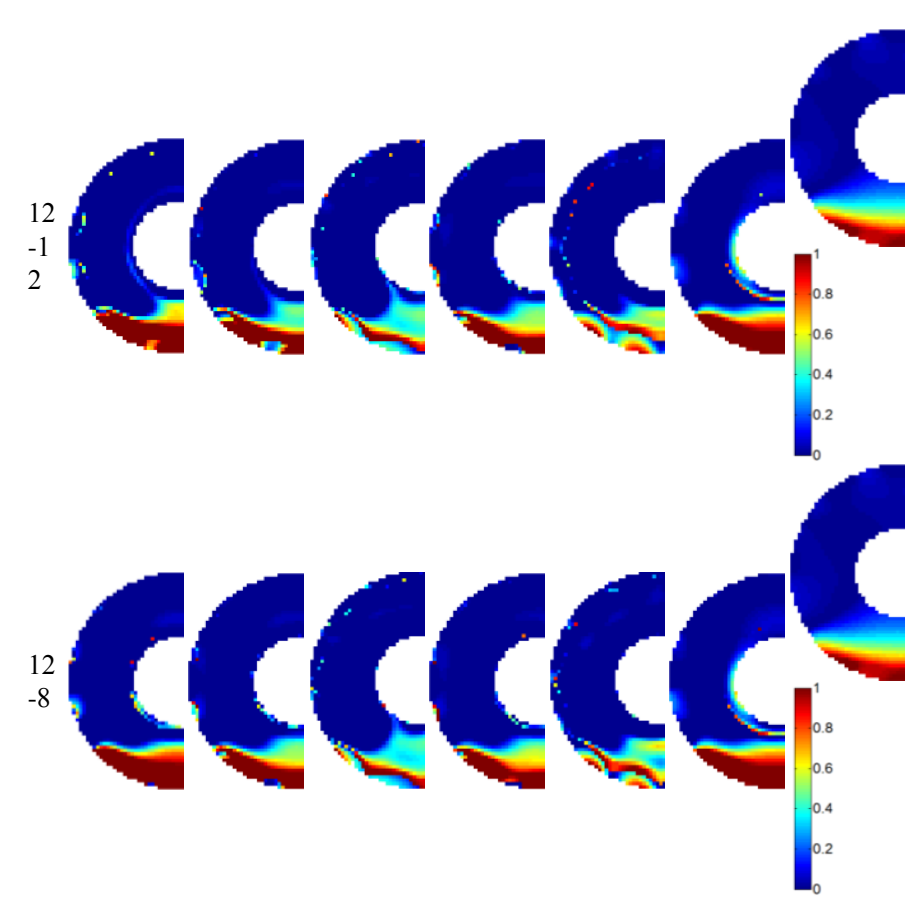



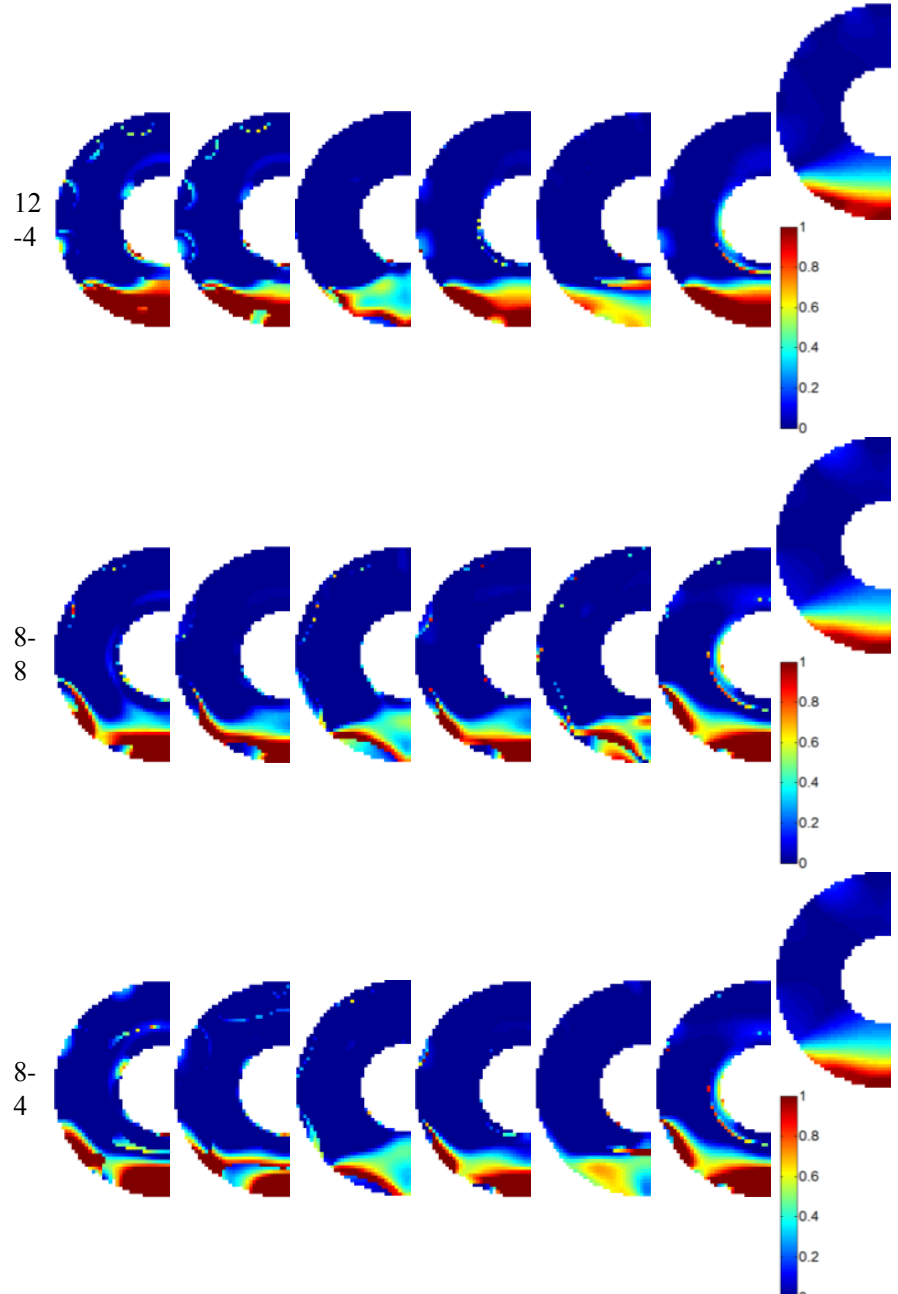

Fig. 6 shows the images reconstructed by the Landweber iteration for stratified distribution 1 . The relative image errors and correlation coefficients between the true distribution and the reconstructed images are given in TABLE VI and TABLE VII, respectively.

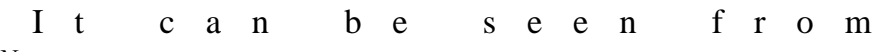
$N_{\mathrm{E}}$

- IEE-EI IEE-EOI IEE-O IEE-EO IEE-OI IEE-E EE $N_{\mathrm{I}}$
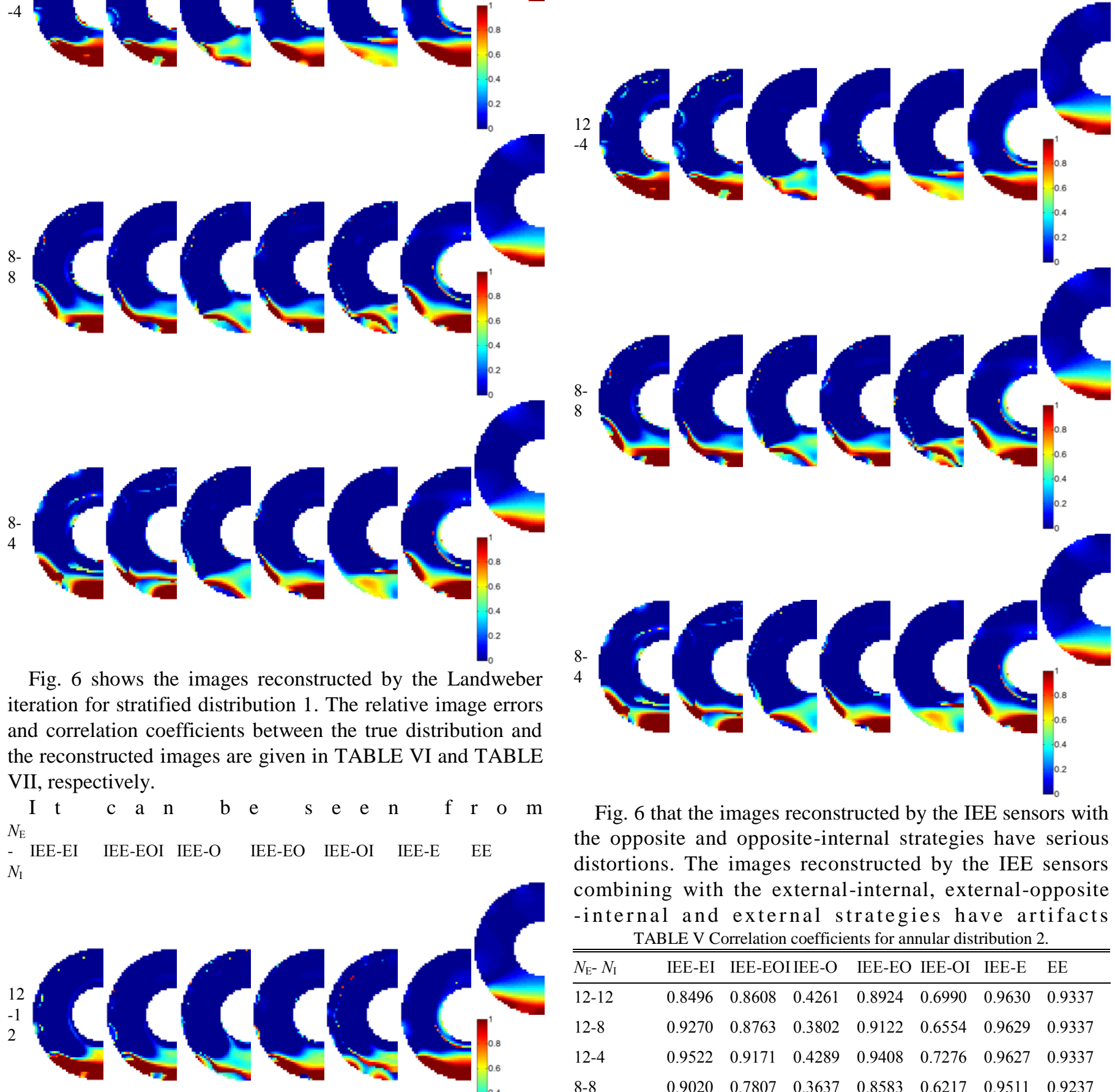

Fig. 6 that the images reconstructed by the IEE sensors with the opposite and opposite-internal strategies have serious distortions. The images reconstructed by the IEE sensors combining with the external-internal, external-opposite -internal and external strategies have artifacts TABLE V Correlation coefficients for annular distribution 2.

\begin{tabular}{llllllll}
\hline \hline$N_{\text {E- }} N_{\mathrm{I}}$ & IEE-EI & IEE-EOI IEE-O & IEE-EO & IEE-OI & IEE-E & EE \\
\hline $12-12$ & 0.8496 & 0.8608 & 0.4261 & 0.8924 & 0.6990 & 0.9630 & 0.9337 \\
$12-8$ & 0.9270 & 0.8763 & 0.3802 & 0.9122 & 0.6554 & 0.9629 & 0.9337 \\
$12-4$ & 0.9522 & 0.9171 & 0.4289 & 0.9408 & 0.7276 & 0.9627 & 0.9337 \\
$8-8$ & 0.9020 & 0.7807 & 0.3637 & 0.8583 & 0.6217 & 0.9511 & 0.9237 \\
$8-4$ & 0.9412 & 0.8166 & 0.4058 & 0.9037 & 0.7615 & 0.9509 & 0.9237 \\
\hline \hline
\end{tabular}

near the internal or external pipe wall.

Comparing the results obtained from the Landweber iteration with IEE and EE sensors quantitatively, it can be seen that the quality of the images reconstructed by the IEE sensor with 12 external electrodes is normally better than that with 8 external electrodes. The image reconstructed by the IEE sensor with 12 
external electrodes and 4 internal electrodes combining with the external-opposite strategy has a lower image error and a higher correlation coefficient than that by the EE sensor with the same number of external electrodes.

In summary, the IEE sensor with 12 external electrodes and 4 internal electrodes combining with the external-opposite strategy gives better image reconstruction for stratified distribution 1.

$N_{\mathrm{E}}$

- IEE-EI IEE-EOI IEE-O IEE-EO IEE-OI IEE-E EE $N_{\mathrm{I}}$

\section{2}
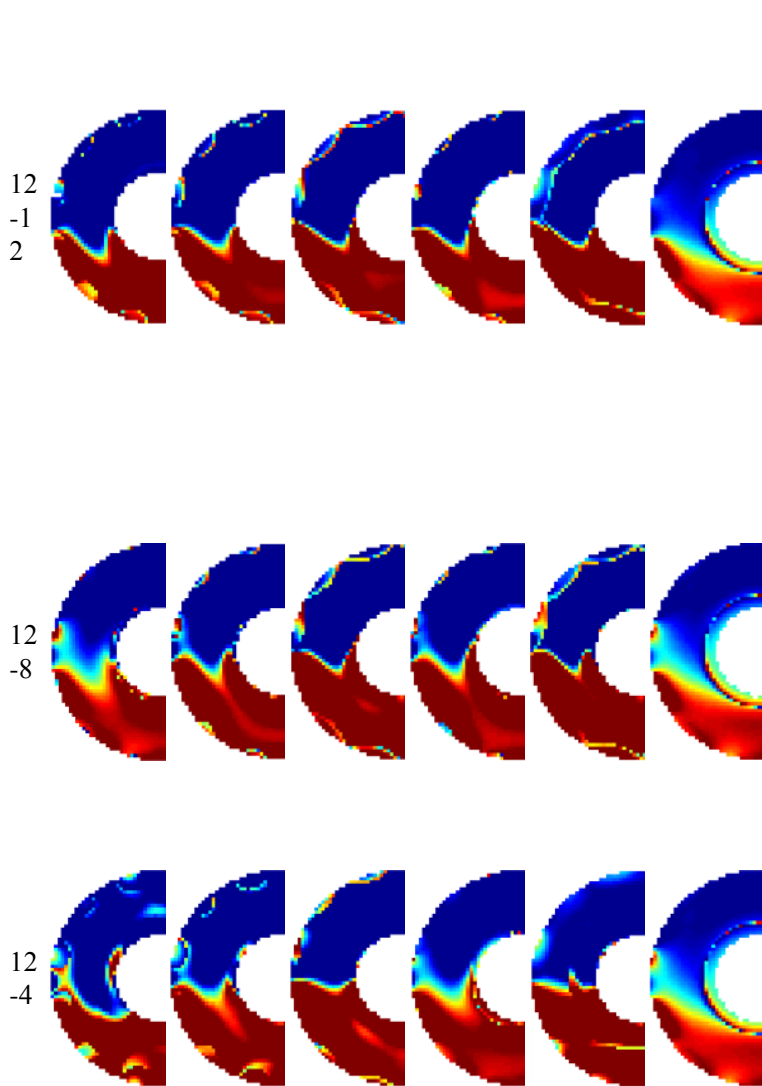

$N_{\text {E- }} N_{\text {I IEE-EI }}$

$12-12$

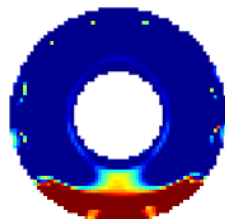

$$
\text { IEE-EOI }
$$

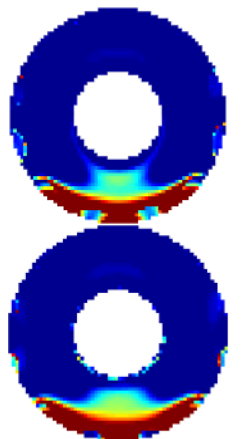

$12-8$
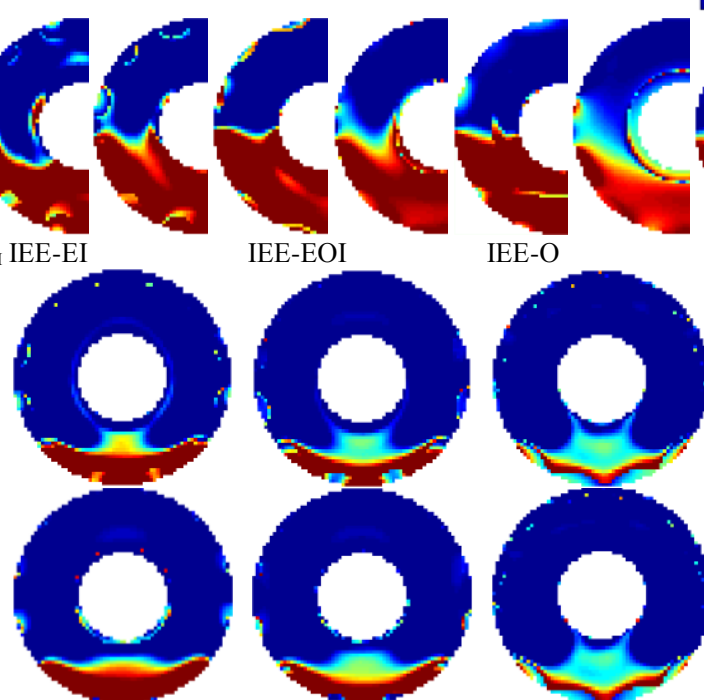

IEE-O

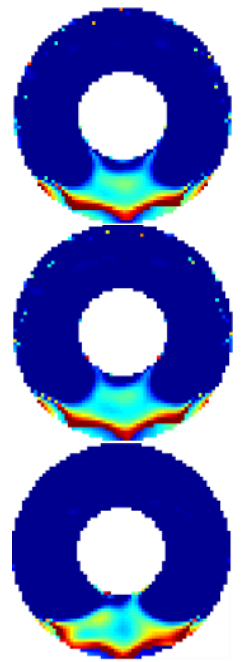

$12-4$
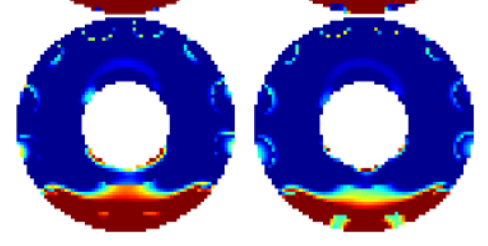

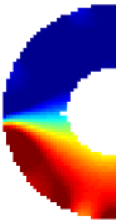

0.8

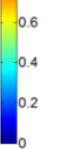

$8-$
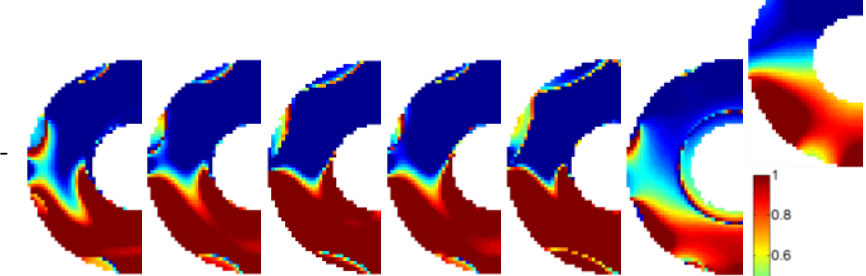

$8-$
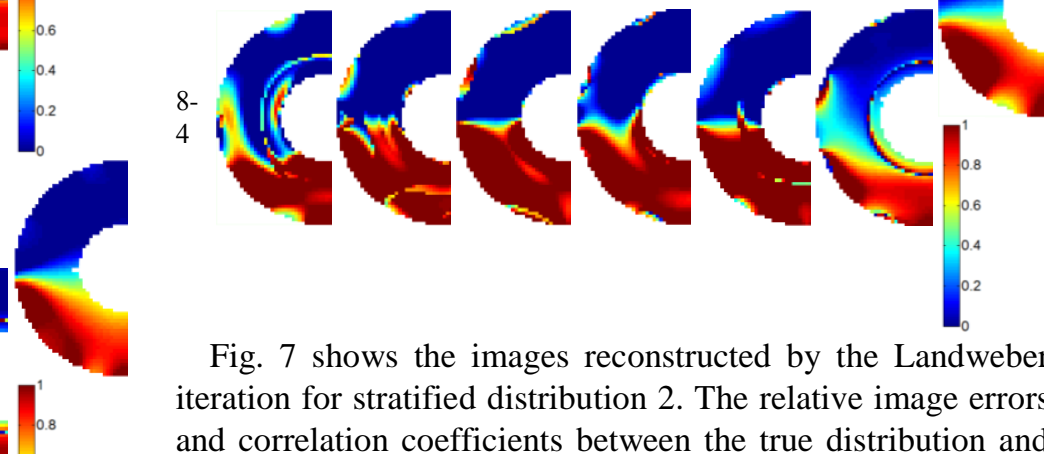

Fig. 7 shows the images reconstructed by the Landweber iteration for stratified distribution 2 . The relative image errors and correlation coefficients between the true distribution and the reconstructed images are given in TABLE VIII and TABLE IX, respectively.
IEE-EO

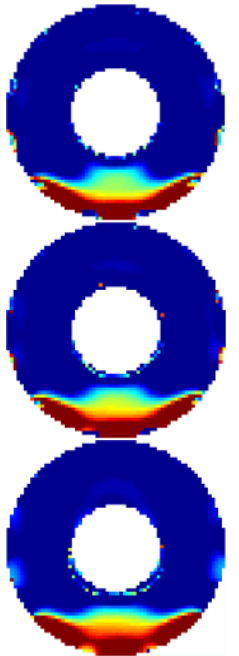

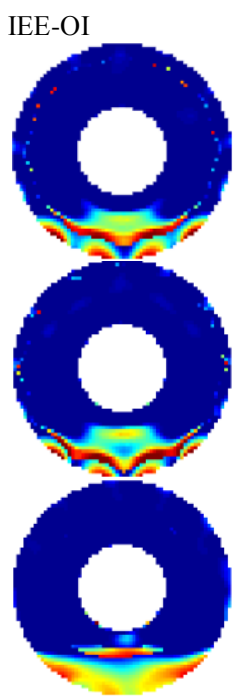

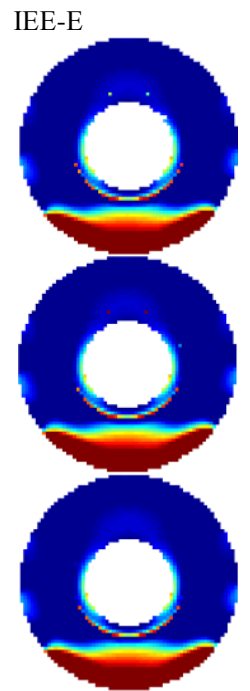

EE

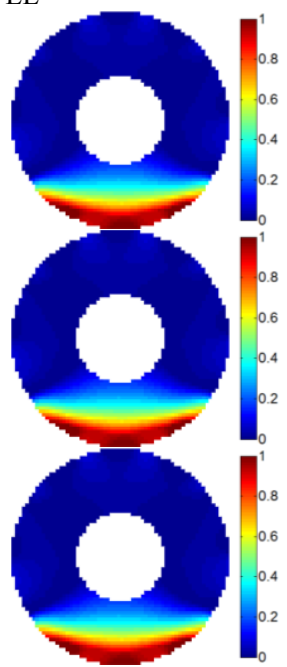



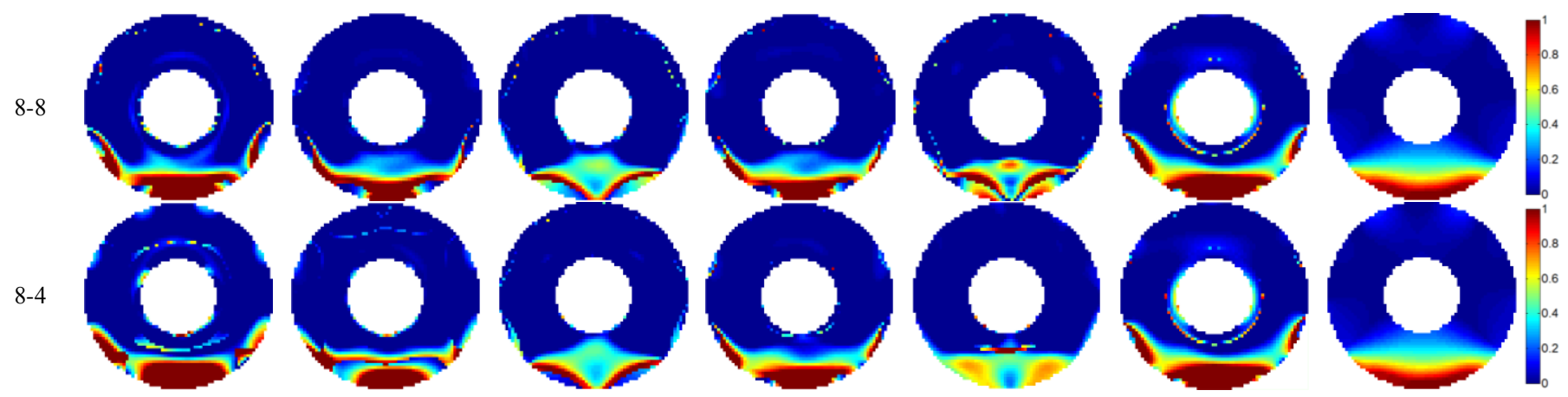

Fig. 6 Images reconstructed for stratified distribution 1.

TABLE VI Image errors for stratified distribution 1.

\begin{tabular}{llllllll}
\hline \hline$N_{\mathrm{E}^{-}} N_{\mathrm{I}}$ & IEE-EI & IEE-EOI IEE-O & IEE-EO & IEE-OI & IEE-E & EE \\
\hline $12-12$ & 0.3974 & 0.4953 & 0.6050 & 0.4736 & 0.5444 & 0.3739 & 0.4112 \\
$12-8$ & 0.3374 & 0.4522 & 0.6109 & 0.4553 & 0.5734 & 0.3723 & 0.4112 \\
$12-4$ & 0.3794 & 0.4049 & 0.5850 & 0.3512 & 0.5600 & 0.3654 & 0.4112 \\
$8-8$ & 0.5820 & 0.5586 & 0.6190 & 0.5479 & 0.5656 & 0.5932 & 0.4348 \\
$8-4$ & 0.6124 & 0.5213 & 0.6190 & 0.5336 & 0.5770 & 0.5963 & 0.4348 \\
\hline \hline
\end{tabular}

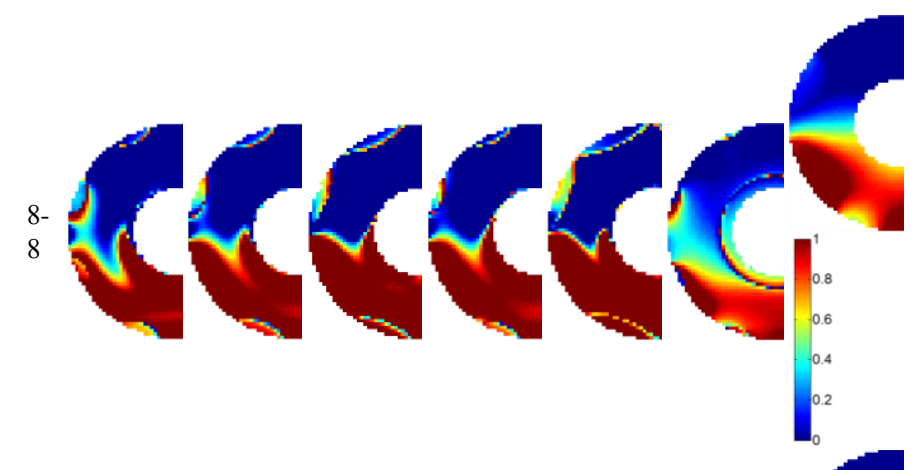

It can be seen from

$N_{\mathrm{E}}$

- IEE-EI IEE-EOI IEE-O IEE-EO IEE-OI IEE-E EE $N_{\text {I }}$
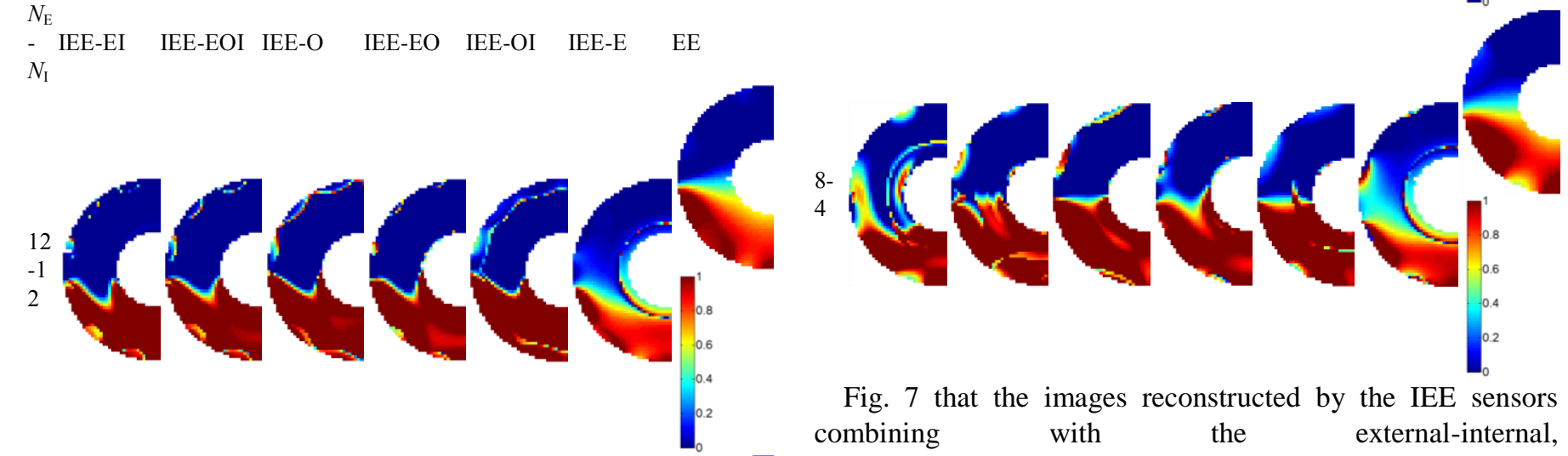

Fig. 7 that the images reconstructed by the IEE sensors combining with the external-internal, external-opposite-internal, opposite, opposite-internal and external strategies have artifacts near the internal or external pipe wall.

Comparing the results obtained from the Landweber iteration with IEE and EE sensors quantitatively, it can be seen that the image reconstructed by the IEE sensor with 12 external electrodes and 4 internal electrodes combining with the external-opposite strategy has the smallest image error.

In summary, the IEE sensor with 12 external electrodes and 4 internal electrodes combining with the external-opposite strategy gives the best image reconstruction for stratified distribution 2 .
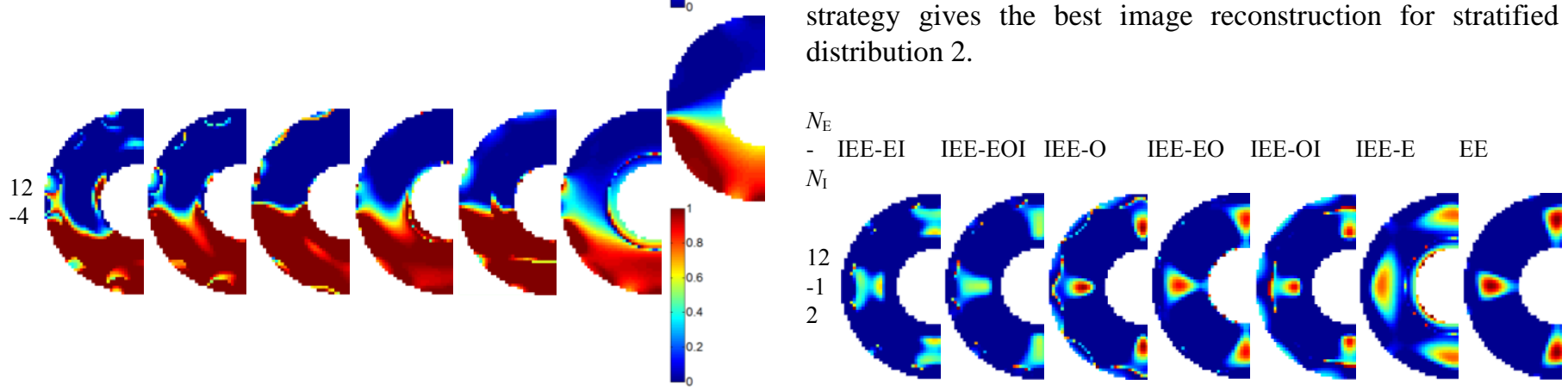


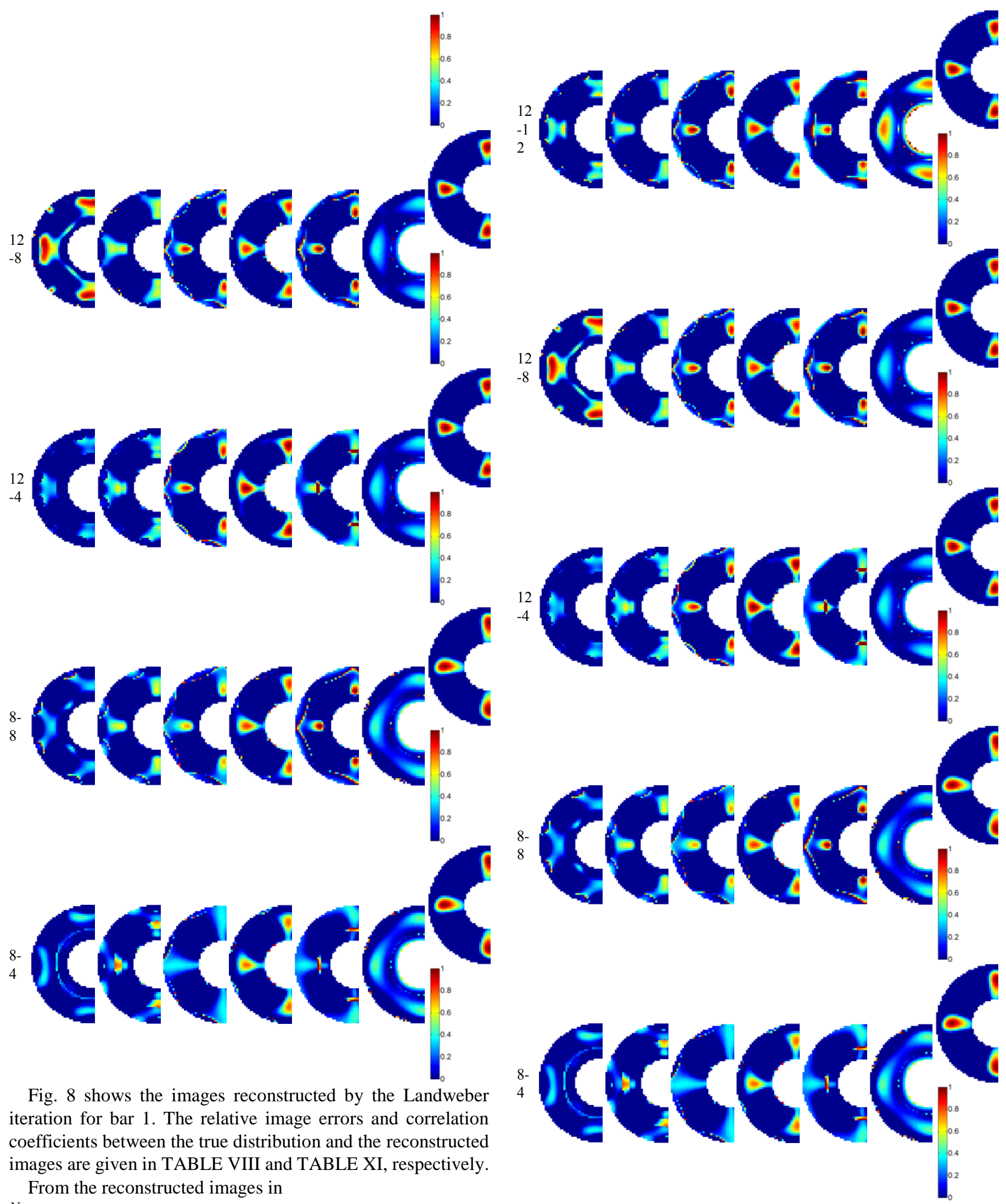

Fig. 8, it can be seen that

- IEE-EI IEE-EOI IEE-O IEE-EO IEE-OI IEE-E EE $N_{\text {I }}$

TABLE VII Correlation coefficients for stratified distribution 1.

\begin{tabular}{llllllll}
\hline \hline$N_{\text {E- }} N_{\mathrm{I}}$ & IEE-EI & IEE-EOI IEE-O & IEE-EO & IEE-OI & IEE-E & EE \\
\hline $12-12$ & 0.8997 & 0.8465 & 0.7934 & 0.8599 & 0.8484 & 0.9131 & 0.9130 \\
\hline
\end{tabular}




\begin{tabular}{llllllll}
\hline \hline $12-8$ & 0.9287 & 0.8725 & 0.7855 & 0.8824 & 0.8157 & 0.9138 & 0.9130 \\
$12-4$ & 0.9105 & 0.8969 & 0.8163 & 0.9278 & 0.8537 & 0.9172 & 0.9130 \\
$8-8$ & 0.7712 & 0.7989 & 0.7851 & 0.8053 & 0.8209 & 0.7677 & 0.9020 \\
$8-4$ & 0.7492 & 0.8256 & 0.7810 & 0.8130 & 0.8476 & 0.7655 & 0.9020 \\
\hline \hline
\end{tabular}

the images reconstructed by the IEE sensors with the external-internal, external-opposite-internal and external strategies have serious distortions. The images reconstructed by the IEE sensors combining with the opposite and opposite-internal strategies have artifacts near the external pipe wall.

Comparing the results obtained from the Landweber iteration with IEE and EE sensors quantitatively, it can be seen that the image error and the correlation coefficient from the IEE sensor combining with the external-opposite strategy are better than that with other strategies. The quality of the image reconstructed by the IEE sensor with 12 external electrodes and 4 internal electrodes combining with the external-opposite strategy is the best in all IEE sensors, although the image error and the correlation coefficient are similar to that from the EE sensor.

$N_{\text {E- }} N_{\mathrm{I}} \quad$ IEE-EI IEE-EOI IEE-O

$12-12$

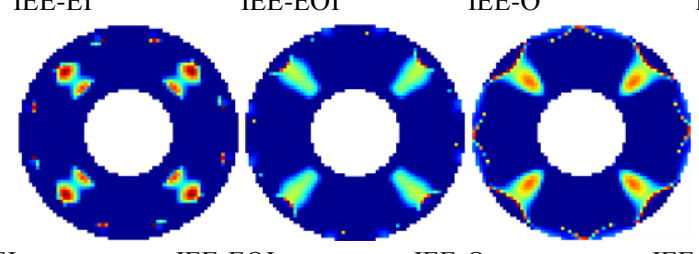

$N_{\text {E- }} N_{\text {I IEE-EI }}$

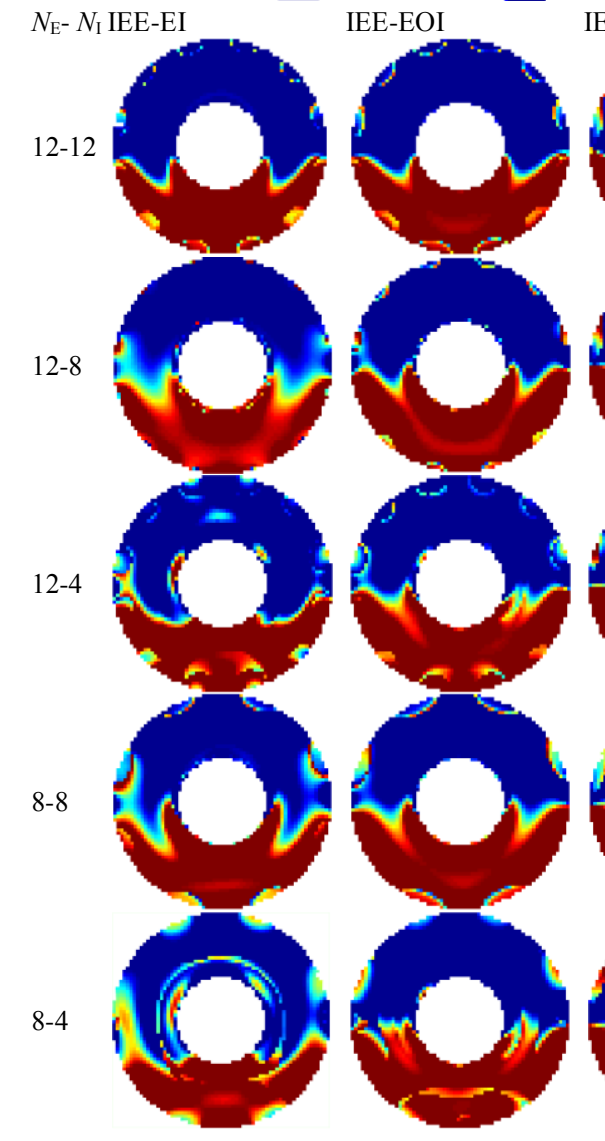

IEE-O

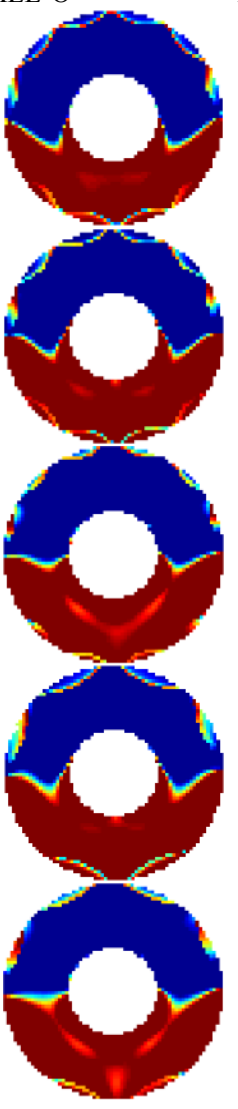

$12-8$

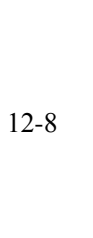

$12-4$

$8-8$
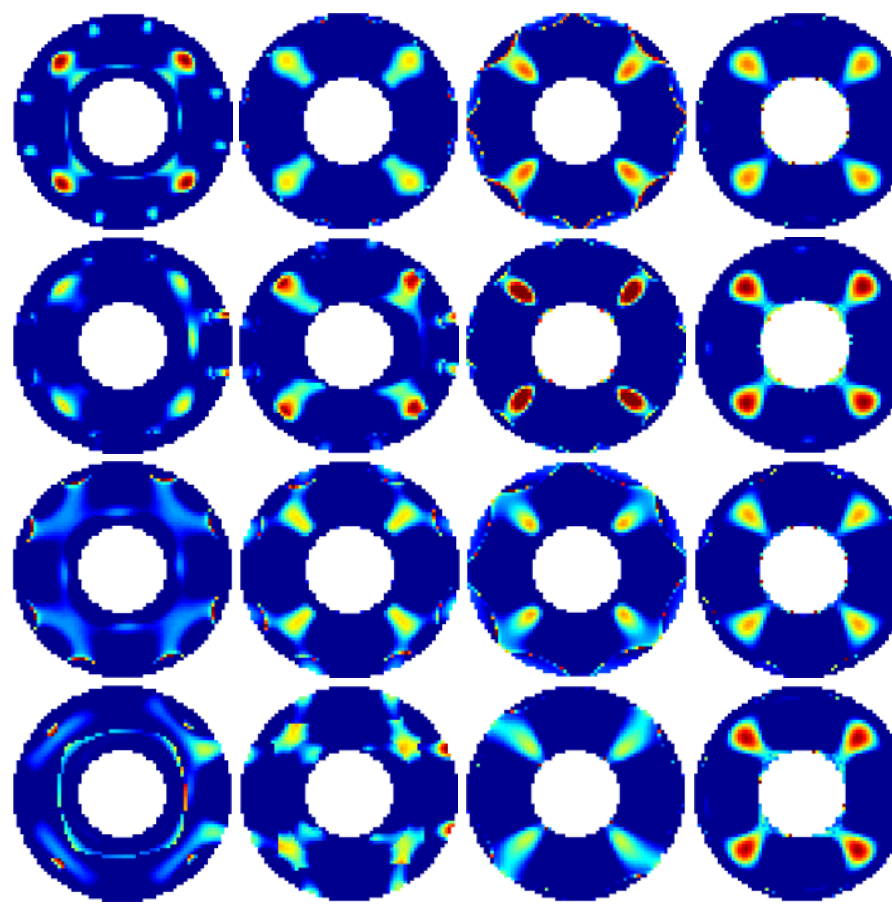

Fig. 9 shows the images reconstructed by the Landweber iteration for bar 2. The relative image errors and correlation
IEE-EO

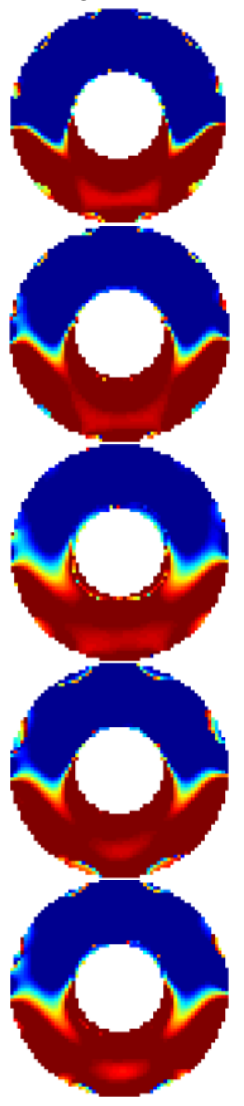

IEE-OI

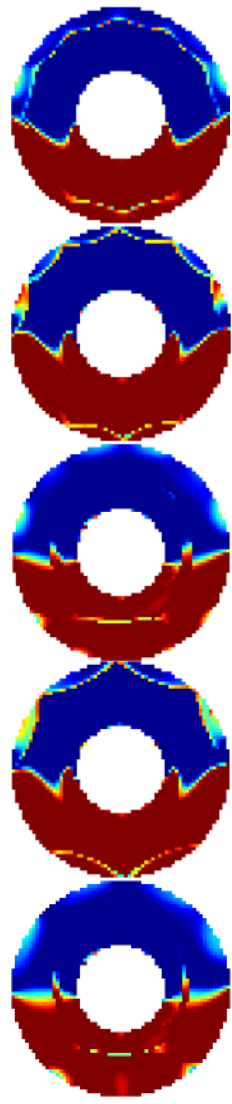

IEE-E

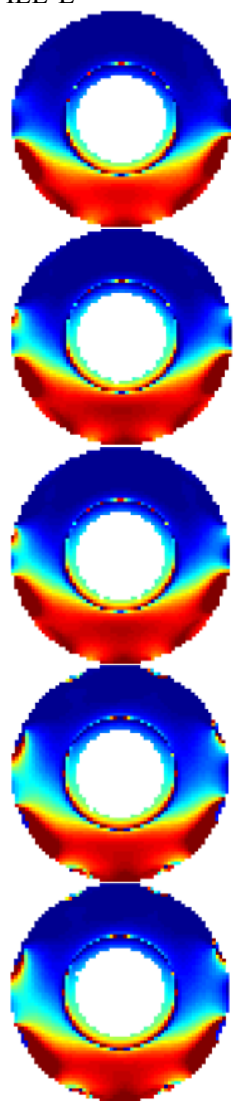

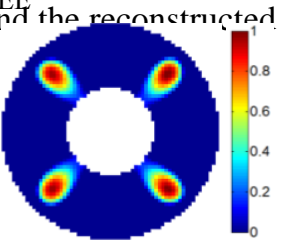

$\mathrm{EE}$

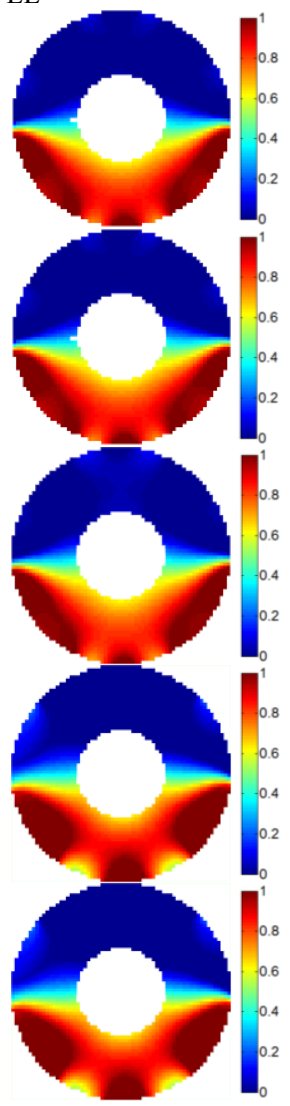


Fig. 7 Images reconstructed for stratified distribution 2.

TABLE VIII Image errors for stratified distribution 2.

\begin{tabular}{llllllll}
\hline \hline$N_{\text {E- }} N_{\text {I }}$ & IEE-EI & IEE-EOI IEE-O & IEE-EO & IEE-OI & IEE-E & EE \\
\hline $12-12$ & 0.4388 & 0.3922 & 0.3930 & 0.3918 & 0.3714 & 0.4427 & 0.2822 \\
$12-8$ & 0.3491 & 0.2963 & 0.3062 & 0.3017 & 0.3009 & 0.4086 & 0.2822 \\
$12-4$ & 0.4879 & 0.2938 & 0.2662 & 0.2751 & 0.2263 & 0.4103 & 0.2822 \\
$8-8$ & 0.4072 & 0.3032 & 0.3075 & 0.3002 & 0.3034 & 0.4773 & 0.2532 \\
$8-4$ & 0.5236 & 0.2906 & 0.2667 & 0.2886 & 0.2351 & 0.4770 & 0.2532 \\
\hline \hline
\end{tabular}

images are given in TABLE XII and TABLE XIII, respectively. sensor. The image quality can be improved if the lower criterion value of the projection operation in Landweber TABLE IX Correlation coefficients for stratified distribution 2.

\begin{tabular}{llllllll}
\hline \hline$N_{\text {E- }} N_{\mathrm{I}}$ & IEE-EI & IEE-EOI IEE-O & IEE-EO & IEE-OI & IEE-E & EE \\
\hline $12-12$ & 0.8185 & 0.8501 & 0.8424 & 0.8504 & 0.8595 & 0.8289 & 0.9404 \\
$12-8$ & 0.8797 & 0.9112 & 0.9032 & 0.9072 & 0.9063 & 0.8425 & 0.9404 \\
$12-4$ & 0.7621 & 0.9138 & 0.9270 & 0.9250 & 0.9475 & 0.8417 & 0.9404 \\
$8-8$ & 0.8262 & 0.9051 & 0.9017 & 0.9072 & 0.9042 & 0.7676 & 0.9494 \\
$8-4$ & 0.7082 & 0.9147 & 0.9255 & 0.9150 & 0.9435 & 0.7682 & 0.9494 \\
\hline \hline
\end{tabular}

It can be seen from

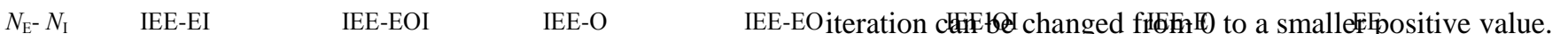

$12-12$
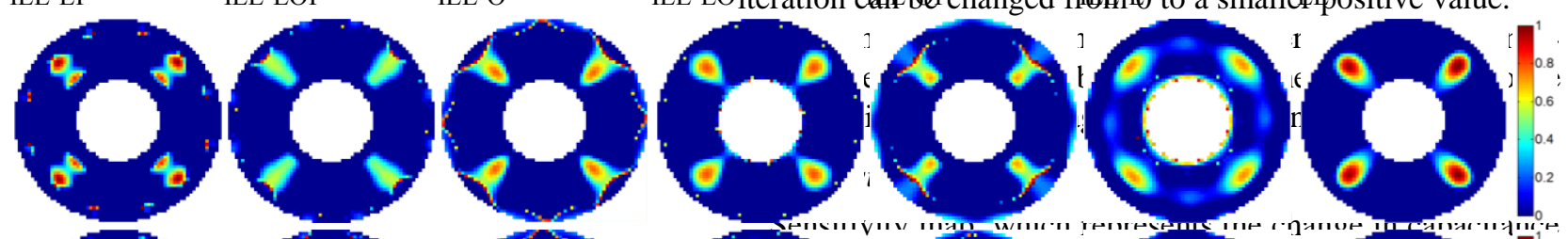

$12-8$
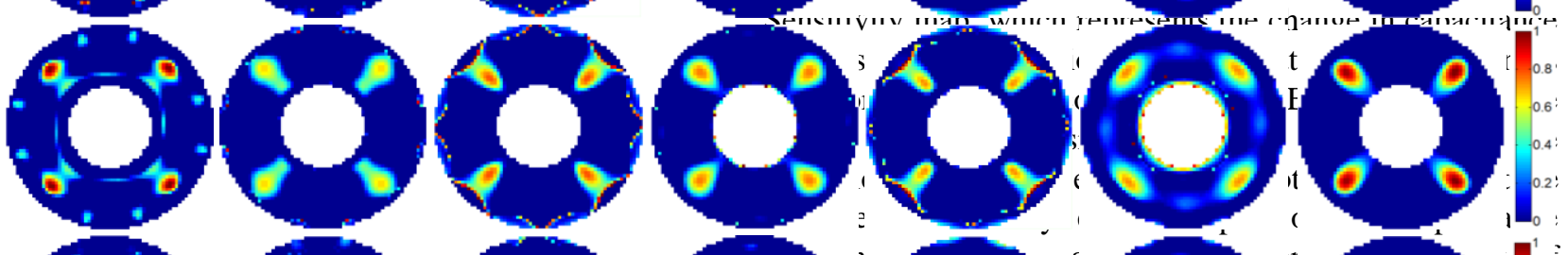

$12-4$
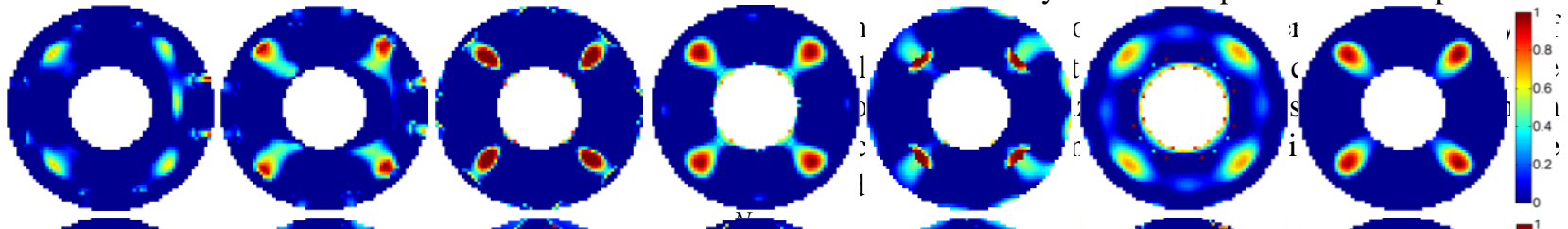

$8-8$
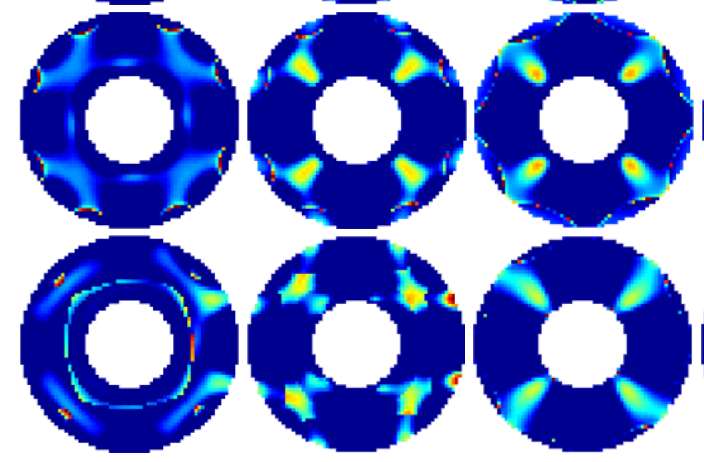

Fig. 9 that the images reconstructed by the IEE sensors with the external-internal, external-opposite- internal, opposite-internal and external strategies have serious distortions. The images reconstructed by the IEE sensors combining with the opposite strategy have artifacts near the external pipe wall.

Comparing the results obtained from the Landweber iteration with IEE and EE sensors quantitatively, it can be seen that the image error and the correlation coefficient obtained from the IEE sensor combining with the external-opposite strategy are better than that with other strategies. The quality of the image reconstructed by the IEE sensor with 12 external electrodes and 4 internal electrodes combining with the external-opposite strategy is the best in all IEE sensors, although the image error and the correlation coefficient are similar to that from the EE
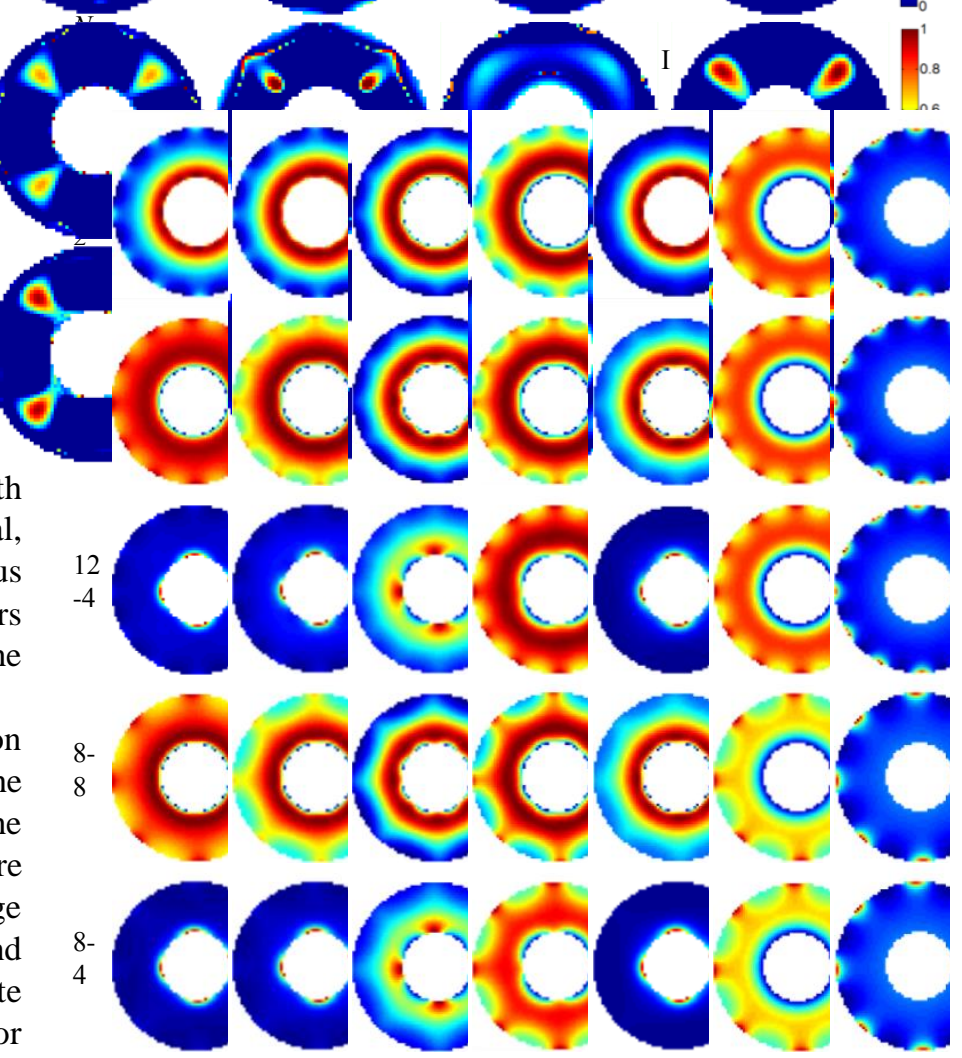

Fig. 10 shows the results.

For the IEE sensors with the external-internal and external- 


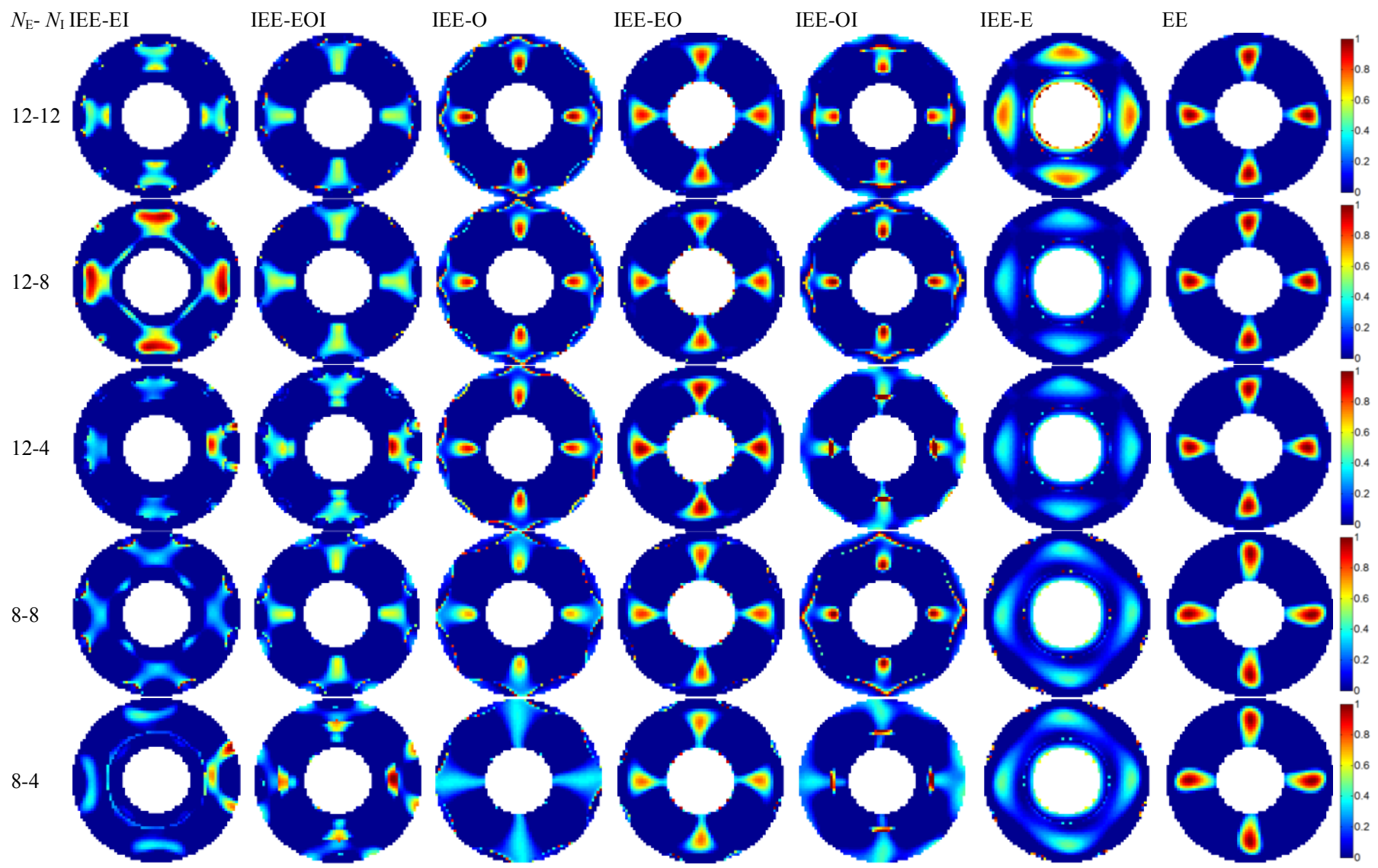

Fig. 8 Images reconstructed for bar 1.

TABLE X Image errors for bar 1.

\begin{tabular}{llllllll}
\hline \hline$N_{\mathrm{E}^{-}} N_{\mathrm{I}}$ & IEE-EI & \multicolumn{2}{l}{ IEE-EOI IEE-O } & IEE-EO & IEE-OI & IEE-E & EE \\
\hline $12-12$ & 0.7665 & 0.7406 & 0.7941 & 0.6627 & 0.8100 & 0.8119 & 0.5618 \\
$12-8$ & 0.7966 & 0.7304 & 0.7937 & 0.6652 & 0.8494 & 0.8264 & 0.5618 \\
$12-4$ & 0.8128 & 0.7626 & 0.8198 & 0.6612 & 0.7718 & 0.8278 & 0.5618 \\
$8-8$ & 0.8885 & 0.7319 & 0.8091 & 0.6805 & 0.8859 & 0.8831 & 0.6304 \\
$8-4$ & 0.9126 & 0.7539 & 0.8501 & 0.6863 & 0.8273 & 0.8772 & 0.6304 \\
\hline \hline
\end{tabular}

opposite-internal strategies, the sensitivity distributions are similar. The sensors with 4 internal electrodes have a higher sensitivity near the internal pipe wall. The sensitivity distribution of this type is only suitable for the image reconstruction of annular distribution. The sensitivity distributions of the other three IEE sensors with externalopposite-internal strategy have a dramatic in-homogeneity, i.e. they are not recommended. Comparing the sensitivity distributions of the other three IEE sensors with external-internal strategy, it can be seen that the IEE sensor with 12 external electrodes and 8 internal electrodes has a more homogenous sensitivity distribution. The quantitative criterion in last section indicated that the IEE sensor with 12 external electrodes and 8 internal electrodes combining with the external-internal strategy can obtain a relative better image quality.

For the IEE sensors with the opposite strategy, the sensitivity distributions vary with the number of external and internal electrodes. The sensitivity distributions of the five IEE sensors
TABLE XI Correlation coefficients for bar 1.

\begin{tabular}{llllllll}
\hline \hline$N_{\text {E- }} N_{\mathrm{I}}$ & IEE-EI & IEE-EOI IEE-O & IEE-EO & IEE-OI & IEE-E & EE \\
\hline $12-12$ & 0.6785 & 0.6609 & 0.5538 & 0.7793 & 0.5235 & 0.5482 & 0.8694 \\
$12-8$ & 0.6725 & 0.7092 & 0.5501 & 0.7749 & 0.4701 & 0.5013 & 0.8694 \\
$12-4$ & 0.5429 & 0.6291 & 0.5143 & 0.8012 & 0.5789 & 0.4976 & 0.8694 \\
$8-8$ & 0.3672 & 0.6555 & 0.5206 & 0.7477 & 0.4171 & 0.3584 & 0.8282 \\
$8-4$ & 0.3170 & 0.6094 & 0.4412 & 0.7430 & 0.4867 & 0.3760 & 0.8282 \\
\hline \hline
\end{tabular}

have a dramatic in-homogeneity, i.e. they are not recommended. The IEE sensors with the opposite-internal strategy have similar sensitivity distributions too. From the reconstructed images and the quantitative analysis, it can be seen that the images reconstructed by these sensors have a very low quality.

For the IEE sensors with the external-opposite strategy, the sensitivity distributions do not have a great deal of difference between the five sensors. Comparing the sensitivity distributions, it can be found that the IEE sensor with 12 external electrodes and 4 internal electrodes has a more homogenous sensitivity distribution than the other four sensors. The sensitivity distribution obtained from this sensor is similar to that obtained from the IEE sensor with 12 external electrodes and 8 internal electrodes combining with the external-internal strategy. The difference is that the IEE sensor with 12 external electrodes and 4 internal electrodes has a higher sensitivity near the internal pipe wall. Combining with the analysis in last section, it can be concluded that the IEE sensor with 12 external electrodes and 4 internal electrodes with the external-opposite 


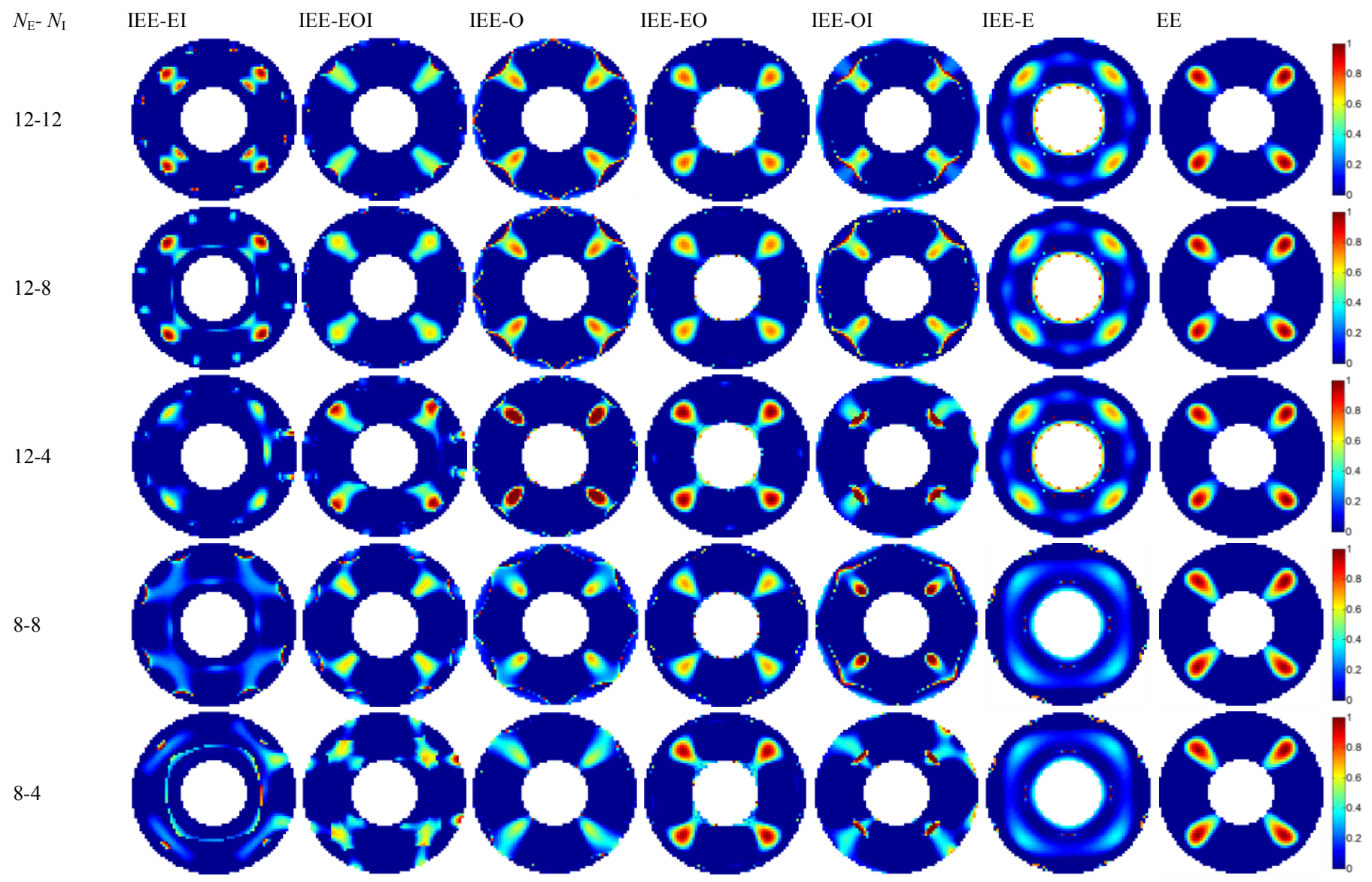

Fig. 9 Images reconstructed for bar 2.

TABLE XII Image errors for bar 2.

\begin{tabular}{llllllll}
\hline \hline$N_{\text {E- }} N_{\mathrm{I}}$ & IEE-EI & IEE-EOI IEE-O & IEE-EO & IEE-OI & IEE-E & EE \\
\hline $12-12$ & 0.7130 & 0.7062 & 0.7610 & 0.6464 & 0.7306 & 0.7597 & 0.5630 \\
$12-8$ & 0.7165 & 0.7037 & 0.7649 & 0.6436 & 0.7892 & 0.7624 & 0.5630 \\
$12-4$ & 0.8025 & 0.7216 & 0.6914 & 0.6372 & 0.6924 & 0.7701 & 0.5630 \\
$8-8$ & 0.9045 & 0.7337 & 0.7869 & 0.6822 & 0.8679 & 0.8925 & 0.6252 \\
$8-4$ & 0.9155 & 0.7555 & 0.7449 & 0.6665 & 0.7799 & 0.8924 & 0.6252 \\
\hline \hline
\end{tabular}

strategy can generate the best images for most distributions.

For the IEE sensors with the external strategy, the sensitivity distribution has a significant difference with the change in the number of external electrodes. The sensitivity distributions have obvious in-homogeneity near the external and internal pipe wall. For some permittivity distributions, the in-homogeneity can result in artifacts in the reconstructed images near the pipe wall.

From

$N_{\mathrm{E}}$

- IEE-EI IEE-EOI IEE-O IEE-EO IEE-OI IEE-E EE $N_{\mathrm{I}}$

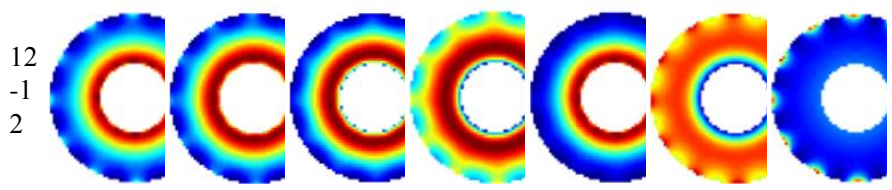

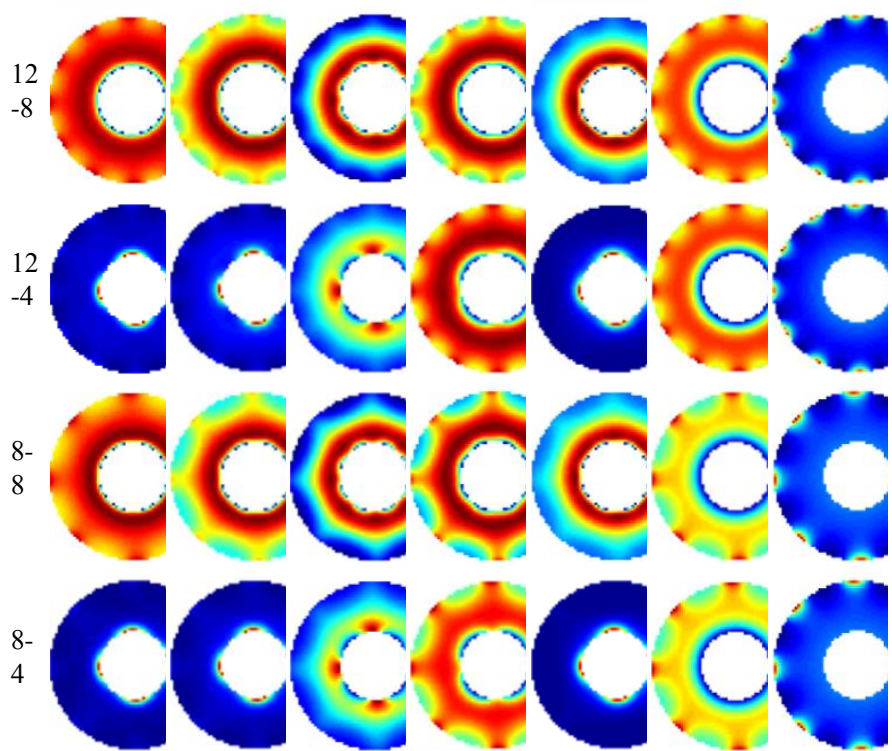

Fig. 10, it can be seen that the averaged sensitivity of EE sensors has a relatively homogenous distribution. However, the sensitivity near the external pipe wall is much higher than that in the central area. For some permittivity distributions, especially the stratified distribution, the images reconstructed by the EE sensor has blurred areas surrounding the phase interface due to the lower sensitivity in the central domain. 
TABLE XIII Correlation coefficients for bar 2.

\begin{tabular}{llllllll}
\hline \hline$N_{\text {E- }} N_{\text {I }}$ & IEE-EI & IEE-EOI IEE-O & IEE-EO & IEE-OI & IEE-E & EE \\
\hline $12-12$ & 0.7254 & 0.7164 & 0.5928 & 0.7851 & 0.6373 & 0.6181 & 0.8801 \\
$12-8$ & 0.7434 & 0.7299 & 0.5874 & 0.7970 & 0.5407 & 0.6105 & 0.8801 \\
$12-4$ & 0.5934 & 0.6911 & 0.6882 & 0.8038 & 0.6872 & 0.5911 & 0.8801 \\
$8-8$ & 0.3233 & 0.6557 & 0.5538 & 0.7508 & 0.4412 & 0.3293 & 0.8446 \\
$8-4$ & 0.2989 & 0.6180 & 0.6504 & 0.8002 & 0.5680 & 0.3299 & 0.8446 \\
\hline \hline
\end{tabular}

\section{IV.CONCLUSION}

In this paper, we discussed the effects of the electrode configuration and the measuring strategy on image $N_{\text {E- }} N_{\mathrm{I}}$ IEE-EI IEE-EOI IEI IEE-O

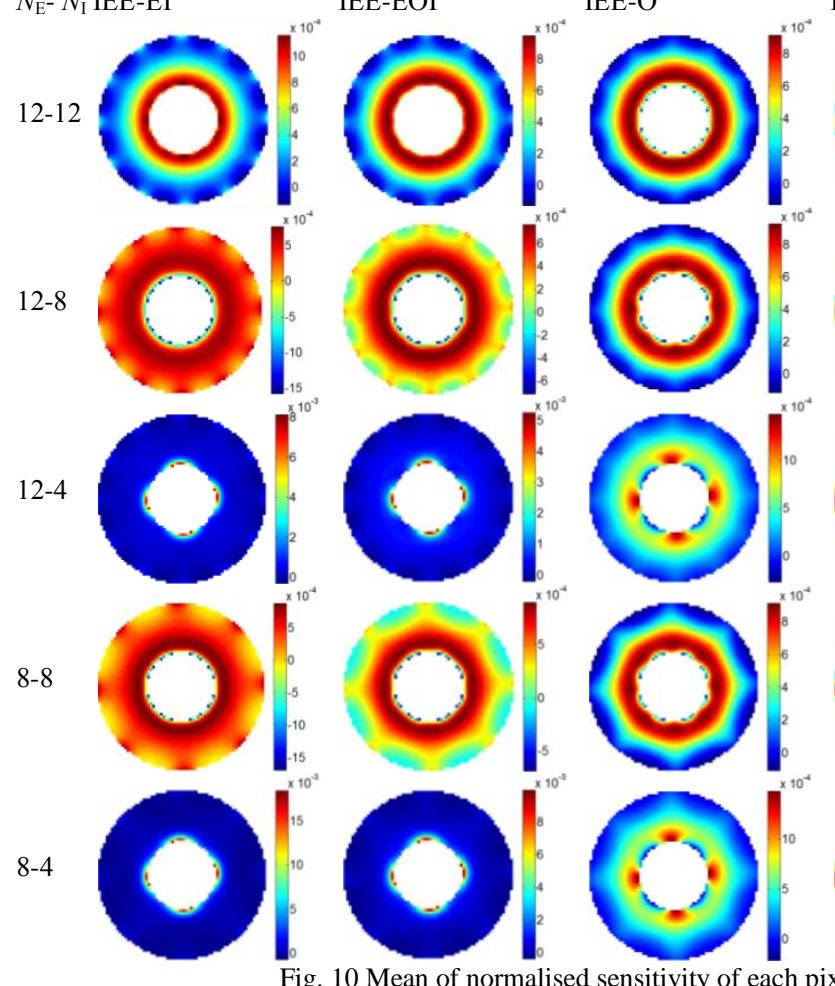

Fig. 10 Mean of normalised sensitivity of each pixel for different combi
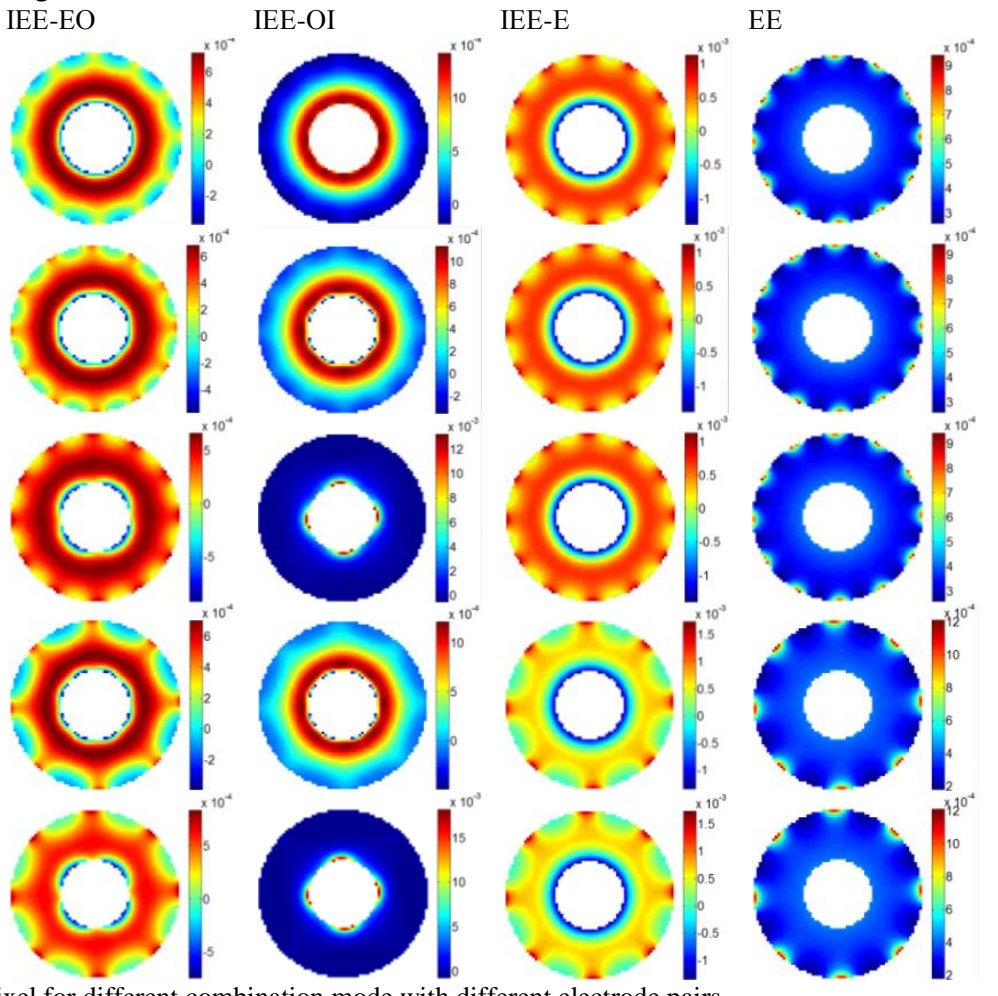

reconstruction with five IEE ECT sensors with different number of internal-external electrodes, 12-12, 12-8, 12-4, 8-8 and 8-4 and two EE ECT sensors with 12 and 8 external electrodes, respectively. Simulation results with Landweber iteration show that the opposite-external strategy is suitable for the IEE ECT sensor to reconstruct the image in a concentric annulus. An IEE ECT sensor with 12 external and 4 internal electrodes combining with the opposite-external strategy is recommended for most applications for the given sensor. Comparing with the traditional EE sensor, the optimal IEE sensor can improve the image quality significantly for most permittivity distributions e.g. annular distribution and stratified distribution.

\section{REFERENCES}

[1] W. Warsito and L.S. Fan, "Measurement of real-time flow structures in gas-liquid and gas-liquid-solid flow systems using electrical capacitance tomography (ECT)", Chem. Eng. Sci., vol. 56, no. 21-22, pp. 6455-6462, 2001.

[2] S. M. Huang, A. B. Plaskowski, C. G. Xie and M. S. Beck, "Tomographic imaging of two-component flow using capacitance sensors," J. Phys. E, Sci. Instrum., vol.22, no.3, pp.173-177, 1989.

[3] G. E. Fasching and N. S. Smith, "A capacitive system for 3-dimensional imaging of fluidized-beds," Rev. Sci. Instrum., vol.62, no.9, pp. 2243-51, 1991.

[4] C. G. Xie, S. M. Huang, B. S. Hoyle, R. Thorn, C. Lenn, D. Snowden and M. S. Beck, "Electrical capacitance tomography for flow imaging system model for development of image reconstruction algorithms and design of primary sensors," IEE Proc.-G, vol.139, pp89-98, Feb. 1992.

[5] W. Q. Yang, "Design of electrical capacitance tomography sensors," Meas. Sci. Technol., vol. 21, no. 4, pp.1-13, Apr. 2010.

[6] S. Liu, W. Q. Yang and H. Wang, "An electrical capacitance tomography sensor with internal-external electrodes," Proc. SPIE, vol. 4188, pp.300 $-307,2001$.

[7] A. Rezvanpour, C. Wang, Y. Liang and W. Q. Yang, "Investigation of droplet distribution in electrohydrodynamic atomization (EHDA) using 
[14] L.H.Peng, H.Merkus and B.Scarlett, "Using regularization methods for image reconstruction of electrical capacitance tomography," Part. Part. Syst. Charact., vol.17, no.3, pp.96-104, 2000.

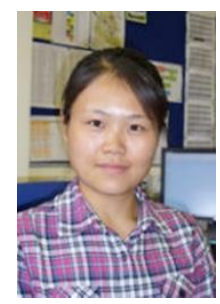

Jiamin Ye received the B.Sc. and M.Sc. degrees in control science and engineering from Tianjin University, Tianjin, China, in 2004 and 2006, respectively, and the $\mathrm{Ph} . \mathrm{D}$. degree in control science and engineering, with a Doctoral thesis on two-phase flow through capacitance sensor from Tianjin University in 2009.

She was a Research Associate with the Department of Automation, Tsinghua University, Beijing, China. Currently, she is a Research Fellow with the School of Process, Environmental and Materials Engineering, University of Leeds, Leeds, UK. Her current research interests include industrial process tomography, image reconstruction, sensing and data acquisition systems, instrumentation and multiphase flow measurement.

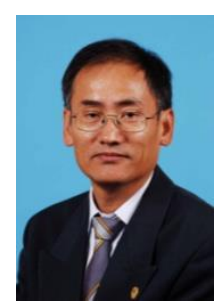

Wuqiang Yang (FIET, FInstMC, FIEEE, CEng) received all his degrees (BEng, $\mathrm{MSc}, \mathrm{PhD}$ ) from Tsinghua University. After 3 years as a Lecturer at Tsinghua University, he joined UMIST in 1991 and currently is a Professor at University of Manchester.

His research is focused on electrical capacitance tomography, with many awards. He has published 300 papers, and holds 10 patents. He is an editorial board member of 5 journals, a guest editor of several journal special issues, and reviews papers for 40 professional journals, including 6 IEEE Transactions. He is also a visiting professor at 6 universities. He has been invited by many universities, research institutions and international conferences worldwide to give lectures, seminars, or keynotes. Since 2002, his biography has been in Who's Who in the World. He is currently an IEEE Instrumentation and Measurement Society Distinguished Lecturer. 